\title{
Automorphisms of the Extended Affine Root System and Modular Property for the Flat Theta Invariants
}

By

\author{
Ikuo SATAKE*
}

\begin{abstract}
We define the central extension $\mathrm{Aut}^{+}(R)$ of the automorphism group $\operatorname{Aut}^{+}(R)$ of the extended affine root system. We give the action of $\tilde{A u t}^{+}(R)$ on the flat theta invariants (theta functions). This describes the modular property for the flat theta invariants.
\end{abstract}

\section{Contents}

$\S 1$ Introduction

$\S 2$ Extended Affine Root System

§3 The Automorphism Group of an Extended Affine Root System

$\S 4$ The Action of $\mathrm{Aut}^{+}(R)$ on the Flat Theta Invariants

\section{§1. Introduction}

(1.1) After K. Saito introduced the simply elliptic singularities in [13], their semi-universal deformations and their period mappings were studied by many people [5], [6], [7], [8], [12], [14], [15], [16]. We have two constructions of the parameter space of the semi-universal deformation. One is geometric, and the other is algebraic (using the extended affine root system). We shall briefly explain these two constructions.

The hypersurface simply elliptic singularities were represented by the following polynomials:

Communicated by K. Saito, August 6, 1992.

1991 Mathematics Subject Classifications: 11F06.

* Department of Mathematics, Faculty of Science, Osaka University, Toyonaka, Osaka 560, Japan. 


$$
\begin{array}{ll}
\tilde{E}_{6}: x(x-z)(x-\lambda z)-z y^{2}=0 & (\lambda \neq 0,1), \\
\tilde{E}_{7}: x y(x-y)(x-\lambda y)-z^{2}=0 & (\lambda \neq 0,1), \\
\tilde{E}_{8}: y\left(y-x^{2}\right)\left(y-\lambda x^{2}\right)-z^{2}=0 & (\lambda \neq 0,1) .
\end{array}
$$

By adding the "lower terms", we have

$$
\begin{aligned}
& \tilde{E}_{6}: x(x-z)(x-\lambda z)-z y^{2} \\
& \quad+a_{1} z+a_{2} x+a_{3} y+a_{4} z x+a_{5} x y+a_{6} z y+a_{7}=0, \\
& \tilde{E}_{7}: x y(x-y)(x-\lambda y)-z^{2} \\
& \quad+a_{1} y+a_{2} x+a_{3} y^{2}+a_{4} x y+a_{5} x^{2}+a_{6} x y^{2}+a_{7} x^{2} y+a_{8}=0, \\
& \tilde{E}_{8}: y\left(y-x^{2}\right)\left(y-\lambda x^{2}\right)-z^{2} \\
& \quad+a_{1} x+a_{2} y+a_{3} x^{2}+a_{4} x y+a_{5} x^{3}+a_{6} x^{2} y+a_{7} x^{4}+a_{8} x^{3} y+a_{9}=0 .
\end{aligned}
$$

According to a criterion [4], [17], this gives the semi-universal deformation. We call the parameter space $\left(\lambda, a_{i}\right)$, the parameter space $S_{0}$ of the semi-universal deformation.

Since a simply elliptic singularity has a unique $\mathbb{C}^{*}$-action, so does its semi-universal deformation space. Therefore the space of the semi-universal deformation is unique up to degree 0 parameter [11]. In this case, the degree 0 parameter is $\lambda$, and $\lambda$ is identified with the value of the elliptic modular function $\lambda(z)$ corresponding to the elliptic curve which appears when the Milnor fiber is compactified.

On the other hand, K. Saito introduced the notion of an extended affine root system, and he reconstructed the parameter space $S$ of the semi-universal deformation as a quotient space of the affine half space $\tilde{\mathbb{E}}$ by the "extended affine Weyl group" $\tilde{W}_{R}$. In this case, the degree 0 parameter is $\tau \in \mathbb{H}$, where $\mathbf{H}$ is a complex affine half space isomorphic to the complex upper half plane.

In order to compare these two constructions, we want to define the $S L(2, \mathbb{Z})$ action on this deformation space $S$. Our main interest is to define the natural $S L(2, \mathbb{Z})$ action on the space $S$.

(1.2) $S L(2, \mathbb{Z})$-actions on $\tilde{\mathbb{E}}$ and $\tilde{W}_{R}$ invariants were already introduced explicitly by Looijenga [7], Kac-Peterson [3] et al. for most types of extended affine root systems. But there exist some types of extended affine root systems on which there is only an action of a subgroup of finite index of $S L(2, \mathbb{Z})$. Also this $S L(2, \mathbb{Z})$ action is not unique. This means that a bigger group which has $S L(2, \mathbb{Z})$ (or its finite index subgroup) as a quotient group, acts on the $\tilde{W}_{R}$ invariants.

In this paper, we introduce the automorphism group of an extended affine root system (in (3.1)), and construct its central extension by using the 
"holomorphic metric" (in (3.5)). This explains why $S L(2, \mathbf{Z})$ acts on the $\tilde{W}_{R}$ invariants, and gives the desired framework of the intrinsic and universal theory of the group which acts on the $\tilde{W}_{R}$ invariants.

(1.3) In [16], certain $\tilde{W}_{R}$ invariants (theta functions) are specified to be the "flat theta invariants". In Theorem 4.7, we give the modular property for these theta functions.

The character for a representation of an affine Kac Moody Lie algebra can be considered as a special $\tilde{W}_{R}$ invariant, therefore our result can be considered to be a framework for the group which acts on these characters.

\section{§ 2. Extended Affine Root System}

We recall some notations from Saito [15], [16]. For details, one is referred to [15], [16].

\section{(2.1) Definition of Extended Affine Root System}

Let $F$ be a real vector space of rank $l+2$ with a positive semi-definite symmetric bilinear form $I: F \times F \rightarrow \mathbb{R}$, whose radical: $\operatorname{rad}(I):=\{x \in F: I(x, y)=$ $0, \forall y \in F\}$, is a vector space of rank 2. For a non-isotropic element $\alpha \in F$ (i.e. $I(\alpha, \alpha) \neq 0)$, put $\alpha^{\vee}:=2 \alpha / I(\alpha, \alpha) \in F$. The reflection $w_{\alpha}$ with respect to $\alpha$ is an element of $O(F, I):=\{g \in G L(F): I(x, y)=I(g(x), g(y))\}$ given by:

$$
w_{\alpha}(u):=u-I\left(u, \alpha^{\vee}\right) \alpha \quad\left({ }^{\forall} u \in F\right) .
$$

Then $\alpha^{\vee} \vee=\alpha$ and $w_{\alpha}^{2}=$ identity.

\section{Definition 2.1.}

1. A set $R$ of non-isotropic elements of $F$ is an extended affine root system belonging to $(F, I)$, if it satisfies the axioms 1)-4):

1) The additive group generated by $R$ in $F$, denoted by $Q(R)$, is a full sub-lattice of $F$. I.e., the embedding $Q(R) \subset F$ induces an isomorphism: $Q(R) \otimes_{\mathbf{Z}} \mathbf{R} \simeq F$.

2) $I\left(\alpha, \beta^{\vee}\right) \in \mathbb{Z}$ for ${ }^{\forall} \alpha, \beta \in R$.

3) $w_{\alpha}(R)=R$ for ${ }^{\forall} \alpha \in R$.

4) If $R=R_{1} \cup R_{2}$ with $R_{1} \perp R_{2}$, then either $R_{1}$ or $R_{2}$ is void.

2. A marking $G$ for the extended affine root system is a rank 1 subspace of $\operatorname{rad}(I)$ such that $G \cap Q(R) \simeq \mathbb{Z}$.

The pair $(R, G)$ will be called a marked extended affine root system. Two marked extended affine root systems are isomorphic if there exists a linear isomorphism of the ambient vector spaces inducing the bijection of the sets of roots and the markings. A generator of $G \cap Q(R) \simeq \mathbb{Z}$, which is unique up 
to a sign, is denoted by $a$.

$$
G \cap Q(R)=\mathbb{Z} a \text { and } G=\mathbb{R} \alpha .
$$

Remark 1. For a root system $R$ belonging to $(F, I)$, there exists a real number $c>0$ such that the bilinear form $c I$ defines an even lattice structure on $Q(R)$ (i.e. $c I(x, x) \in 2 \mathbb{Z}$ for $x \in Q(R)$ ). The smallest such $c$ is denoted by $\left(I_{R}: I\right)$ and the bilinear form $\left(I_{R}: I\right) I$ is denoted by $I_{R}$.

Remark 2. $w_{\alpha}(\alpha)=-\alpha$. Thus the multiplication by -1 is an automorphism of the extended affine root system.

Remark 3. If $u \in \operatorname{rad}(I)$, then $w_{\alpha}(u)=u-I\left(u, \alpha^{\vee}\right) \alpha=u$. Thus the Weyl group acts as the identity on $\operatorname{rad}(I)$.

Remark 4. If $R$ is a root system belonging to $(F, I)$, then $R^{\vee}:=$ $\left\{\alpha^{\vee}: \alpha \in R\right\}$ is also a root system belonging to $(F, I)$.

Remark 5. For a root system $R$ belonging to $(F, I)$, there exists a positive integer $t(R)$ such that

$$
I_{R} \otimes I_{R^{\vee}}=t(R) I \otimes I .
$$

$t(R)$ is called the tier number of $R$.

\section{(2.2) The Basis $\alpha_{0}, \ldots, \alpha_{l}$ for $(R, G)$}

The image of $R$ by the projection $F \rightarrow F / \operatorname{rad}(I)(\operatorname{resp} . F \rightarrow F / G)$ is a finite (resp. affine) root system, which we shall denote by $R_{f}$ (resp. $R_{a}$ ). In this paper, we assume that the affine root system $R_{a}$ is reduced. (I.e. $\alpha=c \beta$ for $\alpha, \beta \in \mathbb{R}_{a}$ and $c \in \mathbb{R}$ implies $c \in\{ \pm 1\}$.)

Once and for all in this paper, we fix $l+1$ elements:

$$
\alpha_{0}, \ldots, \alpha_{l} \in R
$$

such that their images in $R_{a}$ form a basis for $R_{a}$ ([9]). We shall call them a basis for $(R, G)$. Such a basis is unique up to isomorphisms of $(R, G)$. There exist positive integers $n_{0}, \ldots, n_{l}$ such that the sum:

$$
b:=\sum_{i=0}^{l} n_{i} \alpha_{i}
$$

belongs to $\operatorname{rad}(I)$. By a permutation of this basis, we may assume ([9]),

$$
n_{0}=1 \text {. }
$$

Then the images of $\alpha_{1}, \ldots, \alpha_{l}$ in $R_{f}$ form a positive basis for $R_{f}$ and the image of $-\alpha_{0}$ in $R_{f}$ is the highest root with respect to the basis. Put 


$$
L:=\bigoplus_{i=1}^{l} \mathbf{R} \alpha_{i}
$$

on which $I$ is positive definite and $R \cap L$ is a finite root system with the positive basis $\alpha_{1}, \ldots, \alpha_{l}$.

We have a direct sum decomposition of the vector space:

$$
F=L \oplus \operatorname{rad}(I)
$$

and the lattice:

$$
\begin{aligned}
Q(R)=\bigoplus_{i=0}^{l} \mathbf{Z} \alpha_{i} \oplus \mathbf{Z} a & =\bigoplus_{i=1}^{l} \mathbf{Z} \alpha_{i} \oplus \mathbf{Z} a \oplus \mathbf{Z} b, \\
Q(R) \cap \operatorname{rad}(I) & =\mathbf{Z} a \oplus \mathbf{Z} b, \\
Q(R) \cap L & =\bigoplus_{i=1}^{l} \mathbf{Z} \alpha_{i} .
\end{aligned}
$$

Remark. The choice of the basis $\alpha_{0}, \ldots, \alpha_{l}$ is done for the sake of explicit calculation, but it does not affect the result of the present paper. A change of the basis $\alpha_{0}, \ldots, \alpha_{l}$ induces a change $(a, b)$ to $(a, b+m a)$ for some $m \in \mathbb{Z}$.

\section{(2.3) The Weyl Group $W_{R}$}

The Weyl group $W_{R}$ for $R$ is defined as the group generated by the reflections $w_{\alpha}$ for ${ }^{\forall} \alpha \in R$. The projection $p: F \rightarrow F / \operatorname{rad}(I)$ induces a homomorphism $p_{*}: W_{R} \rightarrow W_{R_{f}}$. One gets a short exact sequence:

$$
0 \rightarrow H_{R} \stackrel{E}{\rightarrow} W_{R} \stackrel{p_{*}}{\rightarrow} W_{R_{f}} \rightarrow 1 \text {. }
$$

Here

$$
H_{R}:=\left(\operatorname{rad}(I) \otimes_{\mathrm{R}} F / \operatorname{rad}(I)\right) \cap E^{-1}\left(W_{R}\right)
$$

is a finite index subgroup in the lattice $(\mathbb{Z} a \oplus \mathbf{Z} b) \otimes_{\mathbf{Z}}\left(\oplus_{i=1}^{l} \mathbb{Z} \alpha_{i}^{\vee}\right)$.

The map $E$ called the Eichler-Siegel transformation, is a semi-group homomorphism defined as follows ([15]):

$$
E: F \otimes_{\mathbf{R}} F / \operatorname{rad}(I) \rightarrow \operatorname{End}(F)
$$

$$
E\left(\sum_{i} \xi_{i} \otimes \eta_{i}\right)(u):=u-\sum_{i} \xi_{i} I\left(\eta_{i}, u\right) \quad \text { for } u \in F
$$

Here a semi-group structure $\circ$ on $F \otimes_{\mathbf{R}} F / \operatorname{rad}(I)$ is defined by

$$
\left(\sum_{i} u_{i} \otimes v_{i}\right) \circ\left(\sum_{j} w_{j} \otimes x_{j}\right):=\sum_{i} u_{i} \otimes v_{i}+\sum_{j} w_{j} \otimes x_{j}-\sum_{i, j} I\left(v_{i}, w_{j}\right) u_{i} \otimes x_{j}
$$


The semi-group structure $\circ$ coincides with the natural addition of vectors on the subspace $\operatorname{rad}(I) \otimes(F / \operatorname{rad}(I))$ and hence on $H_{R}$.

\section{(2.4) The Dynkin Graph}

To a marked extended affine root system $(R, G)$, we associate a diagram $\Gamma_{(R, G)}$, called the Dynkin graph for $(R, G)$, in which all data on $(R, G)$ are coded. The graph is constructed in the following steps 1)-4).

1) Let $\Gamma$ be the graph for the affine root system $\left(R_{a}, F / G\right)$, i.e.

a) The set of the vertices $|\Gamma|$ is $\left\{\alpha_{0}, \cdots, \alpha_{l}\right\}$.

b) Edges of $\Gamma$ are given according to a convention in 4) b).

2) The exponent for each vertex $\alpha_{i} \in|\Gamma|$ is defined by

$$
m_{i}:=\frac{I_{R}\left(\alpha_{i}, \alpha_{i}\right)}{2 k\left(\alpha_{i}\right)} n_{i}
$$

where $k(\alpha):=\inf \{n \in \mathbb{N}: \alpha+n a \in R\}$.

3) Put

$$
\begin{aligned}
m_{\max } & :=\max \left\{m_{0}, \cdots, m_{l}\right\}, \\
\left|\Gamma_{m}\right| & :=\left\{\alpha_{i} \in|\Gamma|: m_{i}=m_{\max }\right\}, \\
\left|\Gamma_{m}^{*}\right| & :=\left\{\alpha_{i}+k\left(\alpha_{i}\right) a: \alpha_{i} \in\left|\Gamma_{m}\right|\right\} .
\end{aligned}
$$

4) The graph $\Gamma_{R, G}$ is defined as the graph for $|\Gamma| \cup\left|\Gamma_{m}^{*}\right|$, i.e.

a) The set of the vertices $\left|\Gamma_{R, G}\right|:=|\Gamma| \cup\left|\Gamma_{m}^{*}\right|$.

b) Two vertices $\alpha, \beta \in\left|\Gamma_{R, G}\right|$ are connected by the convention:

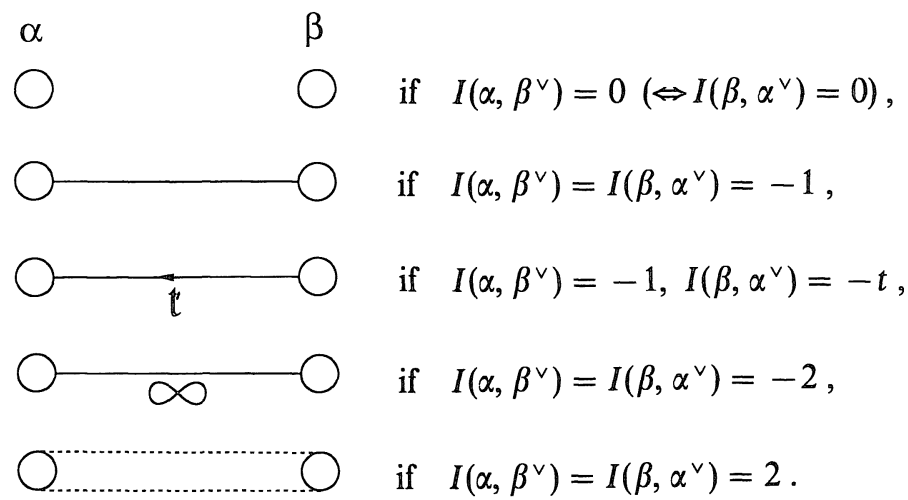

Fig. 1

Definition. For a marked extended affine root $\operatorname{system}(R, G)$, the codimen- 
sion, denoted by $\operatorname{cod}(R, G)$, is defined as follows:

$$
\operatorname{cod}(R, G):=\#\left\{0 \leq i \leq l: m_{i}=m_{\max }\right\}=\#\left|\Gamma_{m}\right| .
$$

Note. The exponents $m_{i}$ 's introduced in 2) are half integers, which might have a common factor. We have:

The smallest common denominator for the rational numbers $m_{i} / m_{\max }(i=$ $0, \ldots, l)$ is equal to $l_{\max }+1([15])$, where $l_{\max }:=\max \{\#$ of vertices in a connected component of $\left.\Gamma \backslash \Gamma_{m}\right\}$.

Thus we sometimes normalize the exponents as follows:

$$
\tilde{m}_{i}:=m_{i} \frac{l_{\max }+1}{m_{\max }} \quad(i=0, \ldots, l) .
$$

\section{(2.5) The Hyperbolic Extension $(\tilde{F}, \tilde{I})$}

There exists a unique (up to a linear isomorphism) real vector space $\tilde{F}$ of rank $l+3$ with

1) an inclusion map $F \subset \tilde{F}$ as a real vector space,

2) a symmetric form $\tilde{I}: \tilde{F} \times \tilde{F} \rightarrow \mathbf{R}$ such that $\left.\tilde{I}\right|_{F}=I$ and $\operatorname{rad}(I)=\mathbf{R} a$.

The pair $(\tilde{F}, \tilde{I})$ will be called a hyperbolic extension for $(F, I)$.

Denote by $\tilde{w}_{\alpha}$ the reflection for $\alpha \in R$ as an element of $G L(\tilde{F})$ and by $\tilde{W}_{R}$ the subgroup of $O(\tilde{F}, \tilde{I})$ (where, $O(\tilde{F}, \tilde{I}):=\{g \in G L(\tilde{F}) \mid \tilde{I}(x, y)=\tilde{I}(g x, g y) \forall x, y \in$ $\tilde{F}\})$ they generate. The restriction $\left.\tilde{w}_{\alpha}\right|_{F}$ is $w_{\alpha}$. Thus we have a surjection $\tilde{W}_{R} \rightarrow W_{R}$ and a short exact sequence:

$$
0 \rightarrow \tilde{K}_{R} \stackrel{\tilde{E}}{\rightarrow} \tilde{W}_{R} \rightarrow W_{R} \rightarrow 1
$$

where $\tilde{K}_{R}$ is an infinite cyclic group generated by

$$
k:=\left(I_{R}: I\right) \frac{l_{\max }+1}{m_{\max }} a \otimes b,
$$

and $\tilde{E}: F \otimes F / G \rightarrow \operatorname{End}(\tilde{F})$ is the Eichler-Siegel transformation:

$$
\tilde{E}\left(\sum_{i} \xi_{i} \otimes \eta_{i}\right)(u):=u-\sum_{i} \xi_{i} \tilde{I}\left(\eta_{i}, u\right) \quad \text { for } u \in \tilde{F}
$$

$\tilde{H}_{R}$ is a subgroup of $\tilde{W}_{R}$ defined as a kernel of the composite map:

$$
\tilde{W}_{R} \rightarrow W_{R} \stackrel{p_{*}}{\rightarrow} W_{R_{f}} .
$$

We have the following diagram: 


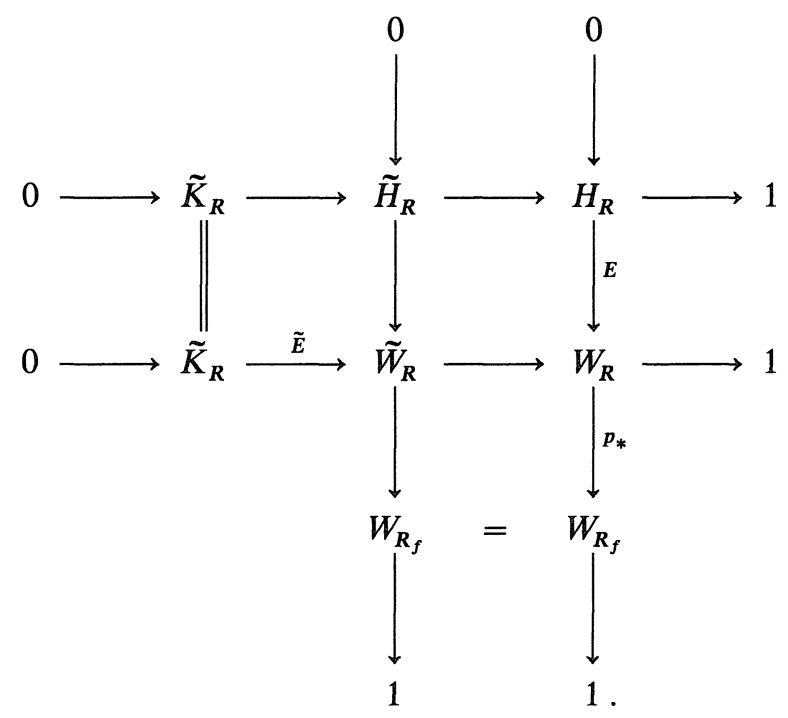

\section{(2.6) A Family of Polarized Abelian Varieties over $\mathbf{H}$}

Let $(R, G)$ be a marked extended affine root system and let $(\tilde{F}, \tilde{I})$ be its hyperbolic extension. We define complex affine half spaces as follows:

$$
\begin{aligned}
& \tilde{\mathbf{E}}:=\left\{x \in \operatorname{Hom}_{\mathbf{R}}(\tilde{F}, \mathbf{C}) ; a(x)=1 \text { and } \operatorname{Im}(b(x))>0\right\}, \\
& \mathbf{E}:=\left\{x \in \operatorname{Hom}_{\mathbf{R}}(F, \mathbf{C}) ; a(x)=1 \text { and } \operatorname{Im}(b(x))>0\right\}, \\
& \mathbf{H}:=\left\{x \in \operatorname{Hom}_{\mathbf{R}}(\operatorname{rad}(I), \mathbf{C}) ; a(x)=1 \text { and } \operatorname{Im}(b(x))>0\right\},
\end{aligned}
$$

where $\operatorname{dim}_{\mathbf{C}} \tilde{\mathbf{E}}=l+2, \operatorname{dim}_{\mathbf{C}} \mathbf{E}=l+1$, and $\operatorname{dim}_{\mathbf{C}} \mathbf{H}=1$. A change of the basis $\alpha_{0}, \ldots, \alpha_{l}$ does not affect the definition of the space $\tilde{E}, \mathbf{E}, \mathbf{H}$. The inclusion maps: $\operatorname{rad}(I) \subset F \subset \tilde{F}$ induce the projections:

$$
\tilde{\mathbf{E}} \stackrel{\tilde{\pi}}{\rightarrow} \mathbf{E} \stackrel{\pi}{\rightarrow} \mathbf{H}
$$

Via the projection, $\tilde{\mathbf{E}}$ and $\mathbf{E}$ are regarded as a total space of a family of complex affine spaces $\tilde{\mathbf{E}}_{x}:=(\pi \circ \tilde{\pi})^{-1}(x)$ and $\mathbf{E}_{x}:=\pi^{-1}(x)$ of dimension $l+1$ and $l$ parametrized by $x \in \mathbf{H}$, and $\tilde{\mathbb{E}}$ has an affine bundle structure on $\mathbf{E}$. The action of the groups $W_{R}$ and $\tilde{W}_{R}$ on $F$ and $\tilde{F}$ fixes $\operatorname{rad}(I)$ pointwise. Hence the contragredient actions of $W_{R}$ and $\tilde{W}_{R}$ induce actions on $\mathbf{E}$ and $\tilde{\mathbf{E}}$ respectively. They are equivarient with the projections $\tilde{\pi}$ and $\pi$ (2.6.4).

Remark. The element $b$ in (2.6.3) induces an isomorphism:

$$
\mathbf{H} \rightarrow \mathscr{H}:=\{z \in \mathbf{C} ; \operatorname{Im}(z)>0\} .
$$

Thus we look on $b$ as a coordinate function on $\mathbf{H}$. When we consider $b$ as 
a coordinate function, we use the letter $\tau$. By (2.2) Remark, $\tau$ is unique up to adding $m \in \mathbf{Z}$.

Lemma 2.1 ([16, p.31]).

1. The actions of $W_{R}$ (resp. $\left.\tilde{W}_{R}\right)$ on $\mathbf{E}$ (resp. $\tilde{\mathbf{E}}$ ) are properly discontinuous.

2. Put $X:=\mathbf{E} / H_{R}$ and denote by $\pi / H_{R}$ the map induced from $\pi$ :

$$
\pi / H_{R}: X \rightarrow \mathbf{H}
$$

The fiber $X_{x}:=\left(\pi / H_{R}\right)^{-1}(x)$ over $x \in \mathbf{H}$ is isogeneous to an l-fold product of elliptic curves of the same modulus $\tau(x)$.

3. The action of $\tilde{H}_{R}$ on $\tilde{\mathbf{E}}$ is fixed point free. Put $L^{*}:=\tilde{\mathbb{E}} / \tilde{H}_{R}$. The map $\tilde{\pi} / \tilde{H}_{R}$ induced from $\tilde{\pi}$ :

$$
\tilde{\pi} / \tilde{H}_{R}: L^{*} \rightarrow X,
$$

defines a principal $\mathbf{C}^{*}$-bundle over $X$. Let $L$ be the associated complex line bundle over $X$, which is, as a set, a union

$$
L=L^{*} \cup X
$$

The finite Weyl group $W_{R_{f}}$ is acting on $L$ and $X$ equivariantly.

4. The Chern class $c\left(L_{X_{x}}\right)$ of the line bundle over $X_{x}:=\left(\pi / H_{R}\right)^{-1}(x)$ for $x \in \mathbf{H}$ is given by

$$
c\left(\left.L\right|_{X_{x}}\right)=\operatorname{Im}(H) \in \bigwedge^{2} \operatorname{Hom}_{\mathbf{Z}}\left(H_{R}, \mathbf{C}\right) \simeq H^{2}\left(X_{x}, \mathbf{C}\right),
$$

where $H$ is the Hermitian form on $V_{\mathbf{C}}=\mathbf{C} \otimes_{\mathbf{R}}(F / \operatorname{rad}(I))^{*}$ given by

$$
H(z, w):=-\frac{m_{\max }}{t(R)\left(l_{\max }+1\right) \operatorname{Im}(\tau(x))} I_{R^{\vee}}(z, \bar{w}) .
$$

\section{(2.7) A Chevalley Type Theorem}

In this subsection, we recall a Chevalley type theorem (Theorem 2.2), studied by Looijenga [6], Schwarzman \& Bernstein [1], [2], Kac \& Peterson [3] and others.

Let us fix a base $\tilde{\lambda} \in \tilde{F} \backslash F$ normalized as

$$
\begin{aligned}
\tilde{I}(\tilde{\lambda}, b) & =1, \\
\tilde{I}\left(\tilde{\lambda}, \alpha_{i}\right) & =0 \quad(1 \leq i \leq l) .
\end{aligned}
$$

Consider this $\tilde{\lambda}$ as a complex coordinate for $\tilde{\mathbf{E}}$, we obtain

$$
(\tilde{\lambda}, \tilde{\pi}): \tilde{\mathbf{E}} \simeq \mathbf{C} \times \mathbf{E} .
$$


The generator $k$ of $\tilde{K}_{R}$ acts on $\tilde{\lambda}$ by

$$
\tilde{E}(k)(\tilde{\lambda})=\tilde{\lambda}-\left(I_{R}: I\right) \frac{l_{\max }+1}{m_{\max }} a .
$$

Hence the complex function $\lambda$ on $\tilde{\mathbf{E}}$ defined by

$$
\lambda:=\exp \left(2 \pi \sqrt{-1} \frac{m_{\max }}{\left(I_{R}: I\right)\left(l_{\max }+1\right)} \tilde{\lambda}\right),
$$

is $\tilde{K}_{R}$ invariant, giving a fiber coordinate for the $\mathbb{C}^{*}$ bundle:

$$
(\lambda, \tilde{\pi}): \tilde{\mathbf{E}} / \tilde{K}_{R} \simeq \mathbf{C}^{*} \times \mathbf{E} .
$$

For a non negative integer $k$, let

$$
S_{k}:=\Gamma\left(X, \mathcal{O}\left(L^{-\otimes k}\right)\right)
$$

be the module of holomorphic sections of the $-k$ th power of the line bundle $L$ over $X$ defined in (2.6) Lemma 2.1. For an element $\Theta \in S_{k}$, put

$$
\tilde{\Theta}:=(\lambda)^{k} \Theta .
$$

Then $\widetilde{\Theta}$ is an $\tilde{H}_{R}$-invariant holomorphic function on $\tilde{\mathbf{E}}$. We sometimes look at an element of $S_{k}$ as a function on $\tilde{\mathbf{E}}$ in this manner. The group $W_{R_{f}} \simeq$ $\tilde{W}_{R} / \tilde{H}_{R}$ acts on $L$ and $X$ equivariantly. Therefore $W_{R_{f}}$ acts on the space of sections $S_{k}(k=0,1, \cdots)$. Put

$$
\begin{aligned}
S_{k}^{W} & :=\text { the set of } W_{R_{f}} \text { invariant elements of } S_{k} . \\
S_{k}^{-W} & :=\text { the set of } W_{R_{f}} \text { anti-invariant elements of } S_{k} . \\
S^{W} & :=\bigoplus_{k=0}^{\infty} S_{k}^{W} . \\
S^{-W} & :=\bigoplus_{k=0}^{\infty} S_{k}^{-W} .
\end{aligned}
$$

Naturally $S^{W}$ is a graded $\Gamma\left(\mathbf{H}, \mathcal{O}_{\mathbf{H}}\right)$-algebra, and the grading is defined by $k$. We prepare one more concept: the Jacobian $J\left(\Theta_{1}, \cdots, \Theta_{l+2}\right)$ for a system of sections $\Theta_{i} \in S_{k_{i}}(i=1, \ldots, l+2)$ as an element of $S_{k}\left(k=\sum_{i=1}^{l+2} k_{i}\right)$ given by the following relation:

$$
d \tilde{\Theta}_{1} \wedge \cdots \wedge d \tilde{\Theta}_{l+2}=\tilde{J}\left(\Theta_{1}, \cdots, \Theta_{l+2}\right)\left(d \tau \wedge d \alpha_{1} \wedge \cdots \wedge d \alpha_{l} \wedge d \tilde{\lambda}\right)
$$

The Jacobian is well defined, since $\omega:=d \tau \wedge d \alpha_{1} \wedge \cdots \wedge d \alpha_{l} \wedge d \tilde{\lambda}$ is $\tilde{H}_{R^{-}}$ invariant. Moreover, since the form $\omega$ is $\tilde{W}_{R}$ anti-invariant and $\Theta_{i} \in S_{k_{i}}^{W}$ $(i=1, \cdots, l+2)$, thus $J\left(\Theta_{1}, \cdots, \Theta_{l+2}\right) \in S_{k}^{-W}\left(k=\sum_{i=1}^{l+2} k_{i}\right)$, (where $\left.\tilde{J}=\lambda^{k} J\right)$. 
Theorem 2.2 ([1][2][6][3]).

1. $S^{W}$ is a polynomial algebra over $\Gamma\left(\mathbf{H}, \mathcal{O}_{\mathbf{H}}\right)$, freely generated by $l+1$ homogeneous elements $\Theta_{0}, \ldots, \Theta_{l}$ of degree $\tilde{m}_{i}:=m_{i} \frac{l_{\max }+1}{m_{\max }}(i=0, \ldots, l)$, where $m_{i}(i=0, \ldots, l)$ is the set of exponents for the root system $(R, G)$.

2. $S^{-W}$ is a free $S^{W}$-module of rank 1 generated by $\Theta_{A}:=J\left(\tau, \Theta_{0}, \cdots, \Theta_{l}\right)$.

Notations. We fix a homogeneous generator system $\Theta_{0}, \ldots, \Theta_{l}$ of the algebra $S^{W}=\Gamma\left(\mathbf{H}, \mathcal{O}_{\mathbf{H}}\right)\left[\Theta_{0}, \ldots, \Theta_{l}\right]$ with an ordering

$$
\operatorname{deg} \Theta_{0} \leq \operatorname{deg} \Theta_{1} \leq \cdots \leq \operatorname{deg} \Theta_{l}=l_{\max }+1
$$

\section{(2.8) The Holomorphic Metric $\tilde{I}_{W}$}

Let us denote by $\mathcal{O}_{\overline{\mathbf{E}}}, \Omega_{\overline{\mathbf{E}}}^{1}$ and $\operatorname{Der}_{\tilde{\mathbf{E}}}$ the sheaf of germs of holomorphic functions, 1-forms and vector fields on $\tilde{\mathbf{E}}$ respectively. Since $\tilde{\mathbf{E}}$ is an open subset of a complex affine space, the tangent and co-tangent spaces of $\tilde{\mathbf{E}}$ are naturally given by:

$$
\begin{aligned}
T_{x}(\tilde{\mathbf{E}}) & \simeq \mathbf{C} \otimes_{\mathbf{R}}(\tilde{F} / G)^{*}, \\
T_{x}^{*}(\tilde{\mathbf{E}}) & \simeq \mathbf{C} \otimes_{\mathbf{R}}(\tilde{F} / G) .
\end{aligned}
$$

Thus we have the canonical isomorphisms:

$$
\Omega_{\tilde{\mathbf{E}}}^{1} \simeq \mathcal{O}_{\tilde{\mathbf{E}}} \otimes_{\mathbf{R}}(\tilde{F} / G) \text { and } \operatorname{Der}_{\tilde{\mathbf{E}}} \simeq \mathcal{O}_{\tilde{\mathbf{E}}} \otimes_{\mathbf{R}}(\tilde{F} / G)^{*} .
$$

The vector space $(\tilde{F} / G)$ carries a nondegenerate symmetric bilinear form induced from $\tilde{I}$. By $\mathcal{O}_{\tilde{\mathbf{E}}}$-bilinear extension of $\tilde{I}$ to $\Omega_{\tilde{\mathbf{E}}}^{1}$, we obtain a form:

$$
\tilde{I}_{\tilde{\mathbf{E}}}: \Omega_{\overline{\mathbf{E}}}^{1} \times \Omega_{\overline{\mathbf{E}}}^{1} \rightarrow \mathcal{O}_{\tilde{\mathbf{E}}}
$$

$$
\omega_{1} \times \omega_{2} \mapsto \sum_{i, j=1}^{l+2} \frac{\omega_{1}}{\partial X_{i}} \frac{\omega_{2}}{\partial X_{j}} \tilde{I}\left(X_{i}, X_{j}\right)
$$

where $X_{i}(i=1, \ldots, l+2)$ are a basis of $\tilde{F} / G$ and $\omega=\sum_{i} \frac{\omega}{\partial X_{i}} d X_{i}$. Since $\tilde{I}_{\tilde{\mathbf{E}}}$ is non-degenerate, the non-degenerate dual form:

$$
\tilde{I}_{\overline{\mathbf{E}}}^{*}: \operatorname{Der}_{\overline{\mathbf{E}}} \times \operatorname{Der}_{\overline{\mathbf{E}}} \rightarrow \mathcal{O}_{\overline{\mathbf{E}}}
$$

is also defined. We call this $\mathcal{O}_{\overline{\mathbf{E}}}$-bilinear form the "holomorphic metric on $\operatorname{Der}_{\tilde{\mathbf{E}}}$."

Put,

(2.8.6) $\operatorname{Der}_{S^{w}}:=$ the module of $\mathbf{C}$-derivations of the algebra $S^{W}$,

$$
\Omega_{S^{w}}^{1}:=\text { the module of } 1 \text {-forms for the algebra } S^{W} .
$$


They are dual $S^{W}$-free modules by the natural pairing $\langle$, > with the dual basis:

$$
\begin{aligned}
\operatorname{Der}_{S^{W}} & =S^{W} \frac{\partial}{\partial \tau} \oplus \bigoplus_{i=0}^{l} S^{W} \frac{\partial}{\partial \Theta_{i}}, \\
\Omega_{S^{W}}^{1} & =S^{W} d \tau \oplus \bigoplus_{i=0}^{l} S^{W} d \Theta_{i},
\end{aligned}
$$

using a generator system of $\Theta_{i}$ 's as in Theorem 2.2. $\operatorname{Der}_{\boldsymbol{S}^{w}}$ and $\Omega_{\mathrm{S}^{w}}^{1}$ have a graded $S^{W}$-module structure in a natural way. There is a natural lifting map:

$$
\begin{aligned}
\Omega_{S^{W}}^{1} & \rightarrow \Omega_{\tilde{\mathbf{E}}}^{1}, \\
d \tilde{\Theta} & \mapsto \sum_{i} \frac{\partial \tilde{\Theta}}{\partial X_{i}} d X_{i},
\end{aligned}
$$

so that the form $\tilde{I}_{\tilde{\mathbf{E}}}$ induces an $S^{W}$-bilinear form:

$$
\tilde{I}_{W}: \Omega_{S^{W}}^{1} \times \Omega_{S^{W}}^{1} \rightarrow S^{W} .
$$

(The values of $\tilde{I}_{W}$ lie in $S^{W}$, since the form $\tilde{I}_{\tilde{\mathbf{E}}}$ is $\tilde{W}_{R}$ invariant.) We call this $S^{W}$-bilinear form "the holomorphic metric on $\Omega_{S^{w}}^{1}$ ". We remark that this symmetric tensor $\tilde{I}_{W} \in \operatorname{Der}_{S^{w}} \otimes \operatorname{Der}_{s^{w}}$ is of degree 0 . Let us denote by $\tilde{I}_{W}^{*}$ the $S^{W}$-bilinear form on the module $\operatorname{Der}_{S^{W}}$ dual to the form $\tilde{I}_{W}$. We use the following Lemma in $\S 4$.

Lemma $([16, \mathrm{p} .36])$.

$$
\tilde{I}_{W}(d \tau, d \tilde{\Theta})=\kappa^{-1} \frac{n}{l_{\max }+1} \tilde{\Theta},
$$

where $\tilde{\Theta} \in S_{n}^{W}$ and $\kappa:=\frac{\left(I_{R}: I\right)}{2 \pi \sqrt{-1} m_{\max }}$.

The proof is easy.

\section{§3. The Automorphism Group of an Extended Affine Root System}

In this section, we define the automorphism group $\operatorname{Aut}^{+}(R)$ of $R$, and its central extension $\mathrm{Aut}^{+}(R)$, which act on $\mathbf{E}$ and $\tilde{\mathbf{E}}$ respectively. Also we show that $\tilde{A u t}^{+}(R)$ contains $\tilde{W}_{R}$ as a normal subgroup.

\section{(3.1) Definition of $\mathrm{Aut}^{+}(\boldsymbol{R})$}

In this subsection, we introduce the automorphism group of $R$.

Definition 3.1. For the extended affine root system $R \subset F$, put Aut $(R):=\{g \in G L(F) \mid g$ induces a bijection of $R\}$. 
Proposition 3.2. The extended affine Weyl group $W_{R}$ is a normal subgroup of Aut $(R)$, and Aut $(R)$ is a subgroup of the orthogonal group $O(F, I)$.

Proof. The latter part follows from Saito [15]. The first part follows from the formula: $g w_{\alpha} g^{-1}=w_{g \alpha}$ for $\alpha \in R, g \in \operatorname{Aut}(R)$.

The element $g$ of the orthogonal group $O(F, I)$ induces a linear transformation of $\operatorname{rad}(I)$ by restriction. We denote this restriction map by $\rho$ :

$$
\rho: O(F, I) \rightarrow G L(\operatorname{rad}(I)),
$$

Definition 3.3. $\Gamma:=\rho($ Aut $(R))$.

$$
\left.g \quad \mapsto g\right|_{\operatorname{rad}(I)} .
$$

Since each $\gamma \in \Gamma$ induces an isomorphism of $Q(R) \cap \operatorname{rad}(I)(\mathbf{Z}$-free module of rank 2), the determinant of $\gamma$ equals \pm 1 . We shall consider only the elements whose determinant equals 1 .

Definition 3.4. We define the following groups:

$$
\begin{aligned}
S L(\operatorname{rad}(I)) & :=\{g \in G L(\operatorname{rad}(I)) \mid \operatorname{det} g=1\}, \\
O^{+}(F, I) & :=\rho^{-1}(S L(\operatorname{rad}(I))), \\
O(F, \operatorname{rad}(I)) & :=\rho^{-1}(1), \\
\Gamma^{+} & :=\Gamma \cap S L(\operatorname{rad}(I)), \\
\operatorname{Aut}^{+}(R) & :=\operatorname{Aut}(R) \cap O^{+}(F, I), \\
\text { Aut }(R, \operatorname{rad}(I)) & :=\text { Aut }(R) \cap O(F, \operatorname{rad}(I)) .
\end{aligned}
$$

The relation between the Weyl group and these groups is given by the following diagram of exact sequences:

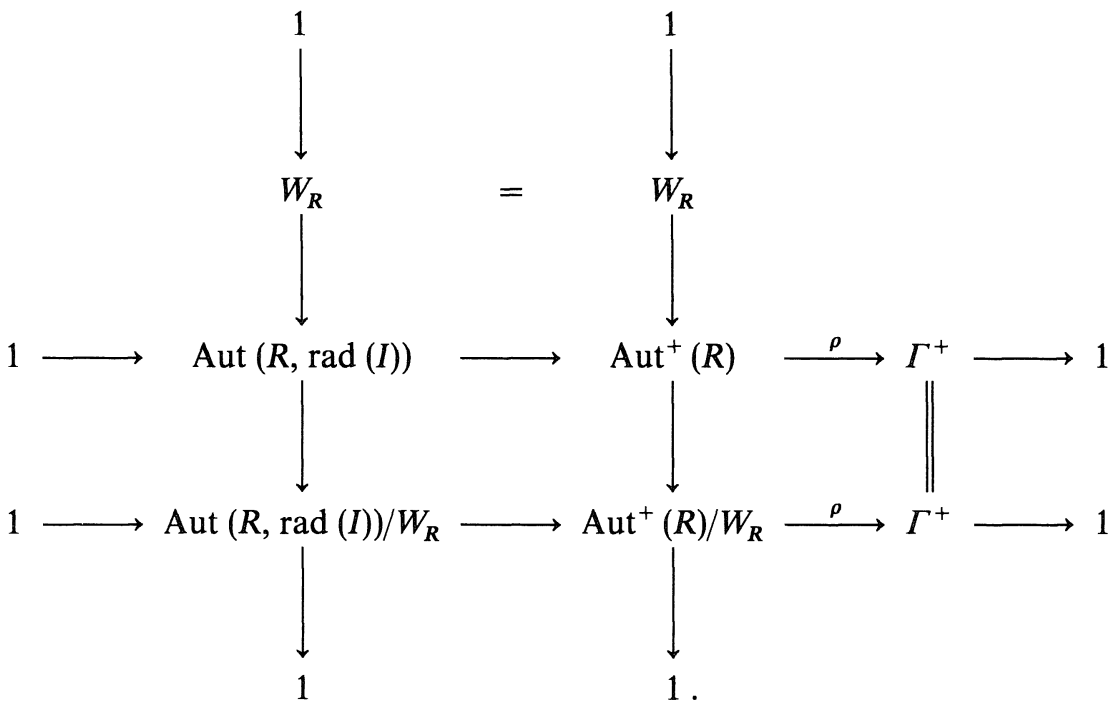




\section{(3.2) The Group Aut $(R, \operatorname{rad}(I)) / W_{R}$}

In this subsection, we assume that $(R, G)$ is reduced and not an exceptional extended affine root system, (i.e. we exclude extended affine root systems of type $A_{1}^{(1,1) *}, B_{l}^{(2,2) *}(l \geq 2), \quad C_{l}^{(1,1) *}(l \geq 2), B C_{l}^{(2,1)}(l \geq 1), B C_{l}^{(2,4)} \quad(l \geq 1)$, $\left.B C_{l}^{(2,2)}(1)(l \geq 2), B C_{l}^{(2,2)}(2)(l \geq 1)\right)$.

(See Saito [15] for these notations.)

For these cases, we give the relation between the group $\operatorname{Aut}(R, \operatorname{rad}(I)) / W_{R}$ and the automorphism groups of the Dynkin graph of a finite and of an affine root system in Proposition 3.5. We fix one $\mathbb{Z}$-basis $a, b \in \operatorname{rad}(I) \cap Q(R)$ such that $\mathbf{Z} a=Q(R) \cap G$. We consider the following commutative diagram:

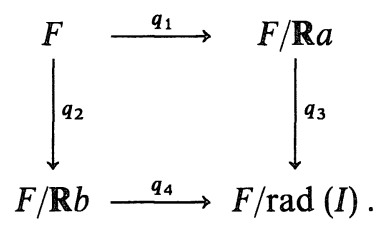

Put

$$
\begin{aligned}
& R_{a}:=q_{1}(R), \\
& R_{b}:=q_{2}(R), \\
& R_{f}:=\left(q_{3} \circ q_{1}\right)(R)\left(=\left(q_{4} \circ q_{2}\right)(R)\right) .
\end{aligned}
$$

$\left(R_{a}\right.$ and $R_{f}$ was already defined in (2.2).)

This defines the affine root systems $\left(F / \mathbf{R} a, R_{a}\right),\left(F / \mathbf{R} b, R_{b}\right)$ and the finite root system $\left(F / \operatorname{rad}(I), R_{f}\right)$. Let $G_{a}$ (resp. $G_{b}$ ) be the automorphism group of an affine Dynkin graph of the root system $R_{a}$ (resp. $R_{b}$ ). Let $G_{f}$ be the automorphism group of a Dynkin graph of the finite root system $R_{f}$. Let $\left(q_{3}\right)_{*}: G_{a} \rightarrow G_{f},\left(q_{4}\right)_{*}: G_{b} \rightarrow G_{f}$ be a natural homomorphism induced by $q_{3}, q_{4}$.

Proposition 3.5. The group Aut $(R, \operatorname{rad}(I)) / W_{R}$ is isomorphic to the group:

$$
\left\{\left(g_{a}, g_{b}\right) \in G_{a} \times G_{b} ;\left(q_{3}\right)_{*}\left(g_{a}\right)=\left(q_{4}\right)_{*}\left(g_{b}\right)\right\}
$$

Proof. An element $g \in$ Aut $(\operatorname{rad}(I))$ induces transformation of $F / \mathbb{R} a, F / \mathbb{R} b$, 
and $F / \operatorname{rad}(I)$. Thus we have the homomorphisms:

$$
\begin{aligned}
&\left(q_{1}\right)_{*}: \text { Aut }(R, \operatorname{rad}(I)) \rightarrow G L(F / \mathbf{R} a), \\
&\left(q_{2}\right)_{*}: \text { Aut }(R, \operatorname{rad}(I)) \rightarrow G L(F / \mathbf{R} b), \\
&\left(q_{3} \circ q_{1}\right)_{*}: \text { Aut }(R, \operatorname{rad}(I)) \rightarrow G L(F / \operatorname{rad}(I)) .
\end{aligned}
$$

Put

$$
\begin{aligned}
& \text { Aut }_{a}:=\left(q_{1}\right)_{*}(\operatorname{Aut}(R, \operatorname{rad}(I))), \\
& \operatorname{Aut}_{b}:=\left(q_{2}\right)_{*}(\operatorname{Aut}(R, \operatorname{rad}(I))), \\
& \operatorname{Aut}_{f}:=\left(q_{3} \circ q_{1}\right)_{*}(\operatorname{Aut}(R, \operatorname{rad}(I))),
\end{aligned}
$$

and

$$
\begin{aligned}
& W_{a}:=\left(q_{1}\right)_{*}\left(W_{R}\right), \\
& W_{b}:=\left(q_{2}\right)_{*}\left(W_{R}\right), \\
& W_{f}:=\left(q_{3} \circ q_{1}\right)_{*}\left(W_{R}\right) .
\end{aligned}
$$

We remark that $\operatorname{ker}\left(q_{1}\right)_{*} \cap \operatorname{ker}\left(q_{2}\right)_{*}=$ identity. Therefore we have the following diagrams:

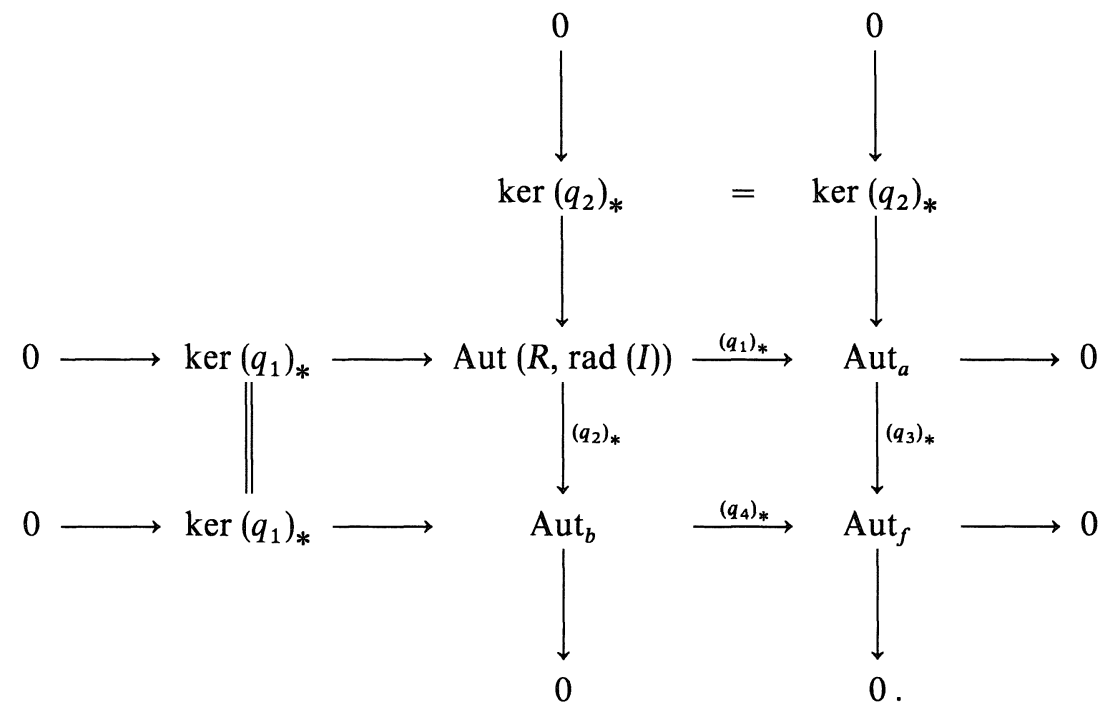


(3.2.15)

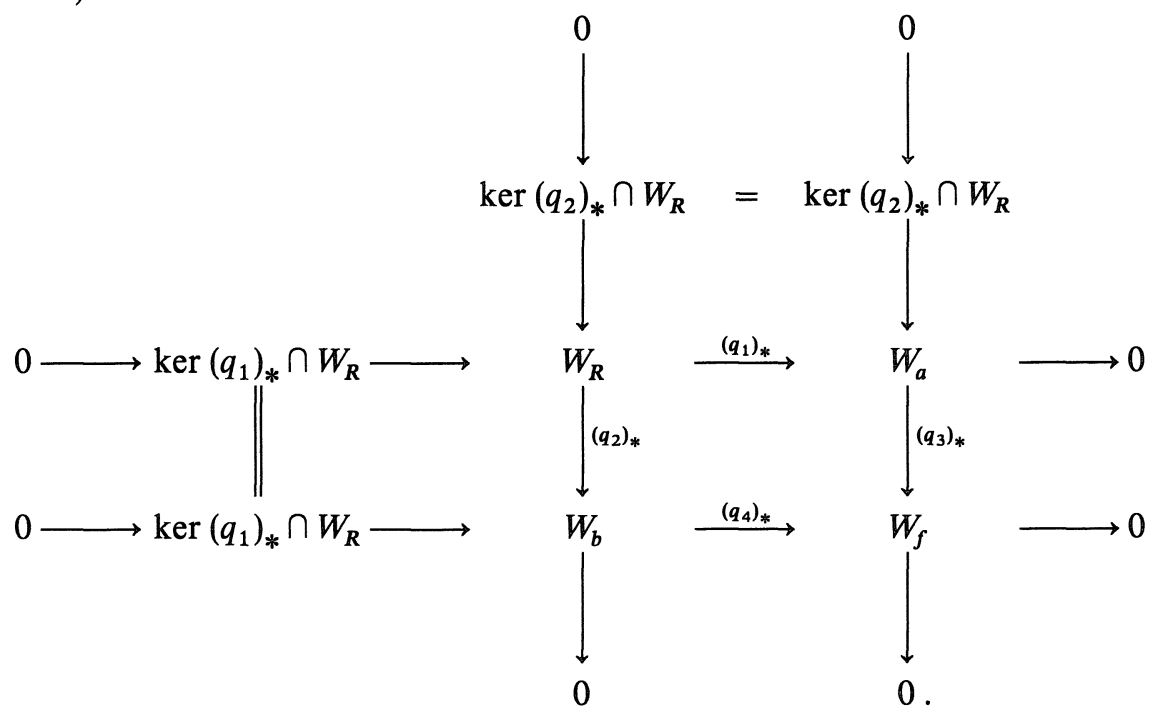

Combining these two diagrams, we have the following diagram:

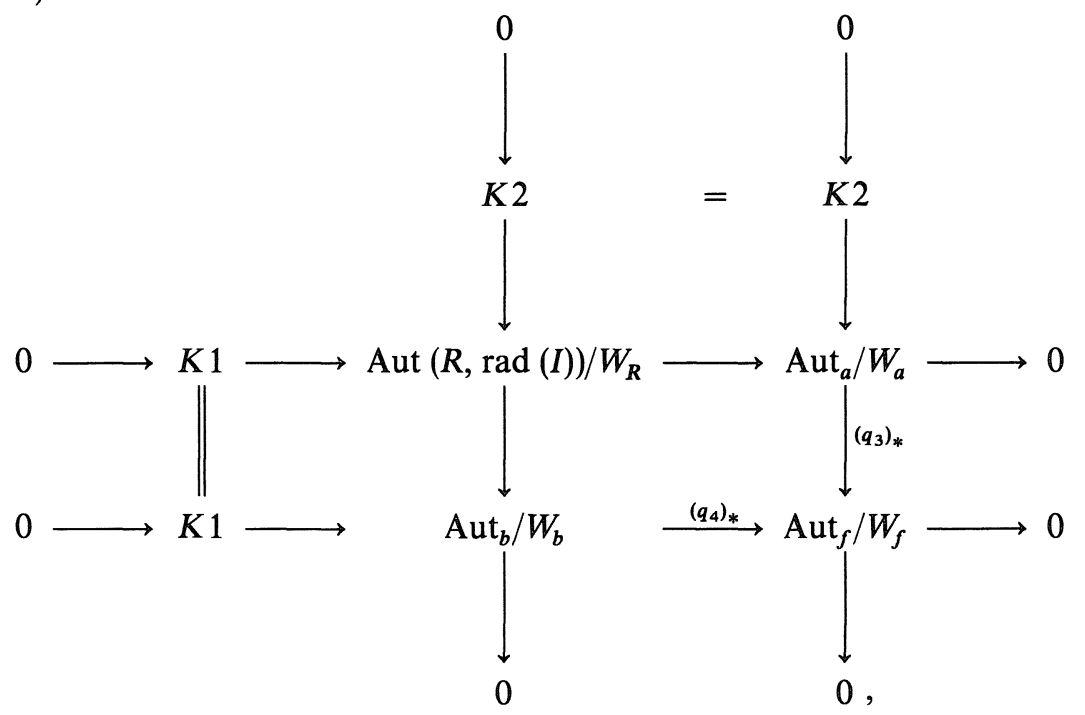

where we write $K 1:=\operatorname{ker}\left(q_{1}\right)_{*} / \operatorname{ker}\left(q_{1}\right)_{*} \cap W_{R}$, and $K 2:=\operatorname{ker}\left(q_{2}\right)_{*} / \operatorname{ker}\left(q_{2}\right)_{*} \cap$ $W_{R}$ in short. The group $\mathrm{Aut}_{a}$ (resp. Aut ${ }_{b}$ ) coincides with the automorphism group of the affine root system $\left(F / \mathbf{R} a, R_{a}\right)$ (resp. $\left(F / \mathbf{R} b, R_{b}\right)$ ) which preserves the null root. The group $W_{a}$ (resp. $W_{b}$ ) coincides with the Weyl group of the affine root system $\left(F / \mathbf{R} a, R_{a}\right)$ (resp. $\left.\left(F / \mathbf{R} b, R_{b}\right)\right)$.

Thus the group $\mathrm{Aut}_{a} / W_{a}$ (resp. Aut ${ }_{b} / W_{b}$ ) coincides with the automorphism 
group $G_{a}$ (resp. $\left.G_{b}\right)$ of the Dynkin graph for the affine root system $\left(F / \mathbf{R} a, R_{a}\right)$ (resp. $\left(F / \mathbf{R} b, R_{b}\right)$ ). Also the group $\mathrm{Aut}_{f} / W_{f}$ coincides with the automorphism group $G_{f}$ of the Dynkin graph for the finite root system $\left(F / \operatorname{rad}(I), R_{f}\right)$.

Therefore the Proposition 3.5 follows from the diagram (3.2.16). Q.E.D.

\section{(3.3) Explicit Description of $\Gamma^{+}$}

We give an explicit description of $\Gamma^{+}$for each marked extended affine root system. Fixing a basis $a, b \in \operatorname{rad}(I) \cap Q(R)(a \in G \cap Q(R))$, we can represent $\Gamma^{+}$as a subgroup of $S L(2, \mathbb{Z})$.

1) $\Gamma^{+}=S L(2, \mathbf{Z})$ for the cases $X_{l}^{(t, t)}, X_{l}^{(t, t) *},(t=1,2,3)$.

2) $\Gamma^{+}=\left\{\left(\begin{array}{ll}p & q \\ r & s\end{array}\right) \in S L(2, \mathbf{Z}) \mid q \equiv 0(\bmod 2)\right\}$ for the cases $B_{l}^{(1,2)}, C_{l}^{(1,2)}$, $F_{4}^{(1,2)}$.

3) $\Gamma^{+}=\left\{\left(\begin{array}{ll}p & q \\ r & s\end{array}\right) \in S L(2, \mathbb{Z}) \mid q \equiv 0(\bmod 3)\right\}$ for the case $G_{2}^{(1,3)}$.

4) $\Gamma^{+}=\left\{\left(\begin{array}{ll}p & q \\ r & s\end{array}\right) \in S L(2, \mathbf{Z}) \mid r \equiv 0(\bmod 2)\right\}$ for the cases $B_{l}^{(2,1)}, C_{l}^{(2,1)}$, $F_{4}^{(2,1)}, B C_{l}^{(2,4)}, B C_{l}^{(2,2)}(2)$.

5) $\Gamma^{+}=\left\{\left(\begin{array}{ll}p & q \\ r & s\end{array}\right) \in S L(2, \mathbf{Z}) \mid r \equiv 0(\bmod 3)\right\}$ for the case $G_{2}^{(3,1)}$.

6) $\Gamma^{+}=\left\{\left(\begin{array}{ll}p & q \\ r & s\end{array}\right) \in S L(2, \mathbb{Z}) \mid p \equiv 1(\bmod 2), q \equiv 0(\bmod 2)\right\}$ for the cases $B C_{l}^{(2,1)}, B C_{l}^{(2,2)}(1)$.

\section{(3.4) The Action of $\mathrm{Aut}^{+}(\boldsymbol{R})$ on $\mathbf{E}$}

In order to define the action of $\operatorname{Aut}^{+}(R)$ on the space $\mathbf{E}$, we introduce the space $F_{\text {half }}^{*}$ as follows:

$$
F_{\text {half }}^{*}:=\left\{x \in \operatorname{Hom}_{\mathbf{R}}(F, \mathbf{C}) \mid\langle a, x\rangle \neq 0,\langle b, x\rangle \neq 0, \operatorname{Im} \frac{\langle b, x\rangle}{\langle a, x\rangle}>0\right\}
$$

The space $F_{\text {half }}^{*}$ has a $C^{*}$ action induced from the $\mathbf{C}^{*}$ action on the complex vector space $\operatorname{Hom}_{\mathbf{R}}(F, \mathbf{C})$, defined by

$$
(\alpha f)(x):=\alpha(f(x)) \quad \text { for } f \in \operatorname{Hom}_{\mathbf{R}}(F, \mathbf{C}), \quad x \in F, \alpha \in \mathbf{C}^{*} .
$$

We consider the following diagram:

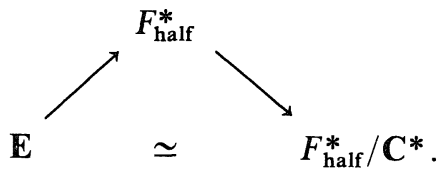


The composite map of the $\mathbf{C}^{*}$ quotient map and the natural inclusion $\mathbb{E} \hookrightarrow F_{\text {half }}^{*}$ becomes an isomorphism. $O^{+}(F, I)$ acts on $F_{\text {half }}^{*}$ contragrediently, thus $O^{+}(F, I)$ also acts on $F_{\text {half }}^{*} / \mathbb{C}^{*}$. Using the above isomorphism (3.4.2), we can define the action of $\mathrm{O}^{+}(F, I)$ and its subgroup $\operatorname{Aut}^{+}(R)$ on $\mathbf{E}$. (We call this action "the linear fractional transformation".)

Remark. Among the element of $\mathrm{O}^{+}(F, I)$, only \pm 1 can be considered as a $\mathbb{C}^{*}$-action. Therefore, $O^{+}(F, I) /\{ \pm 1\}$ acts on the space $\mathbb{E}$ faithfully. The subgroup $O(F, \operatorname{rad}(I))$ does not contain -id., hence $O(F, \operatorname{rad}(I))$ acts on the space $\mathbf{E}$ faithfully. We have the following diagram:

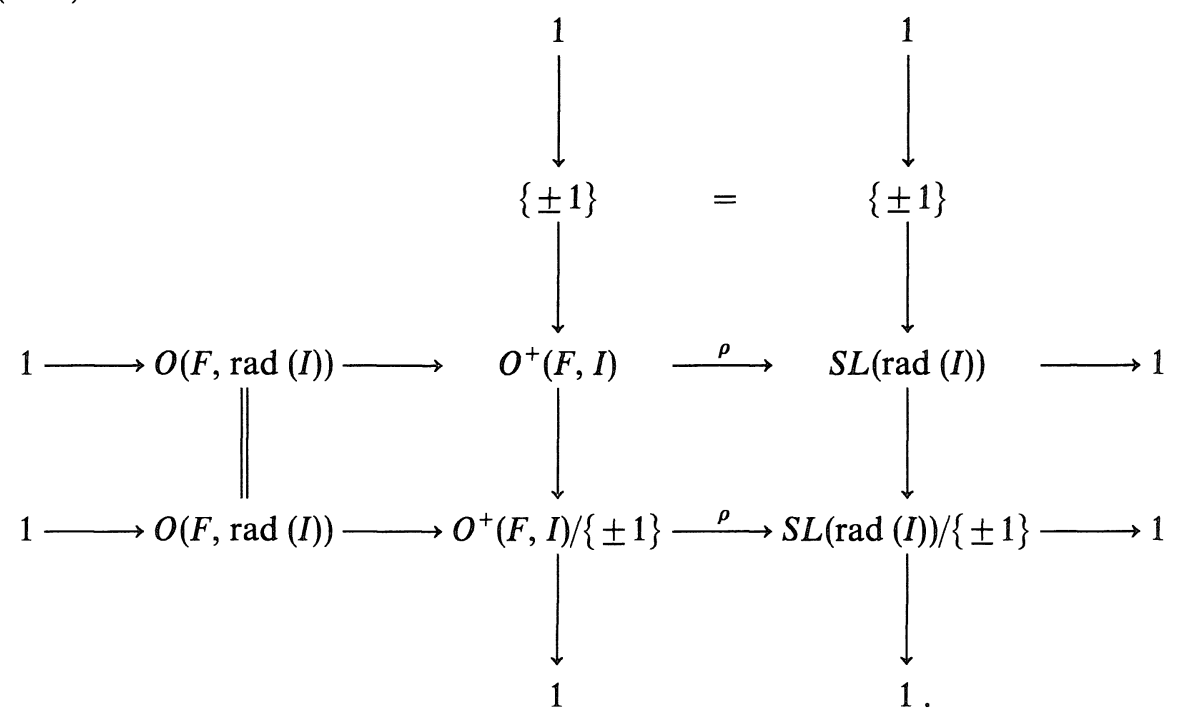

$O^{+}(F, I) /\{ \pm 1\}$ acts on $\mathbb{E}$ faithfully and transitively, and $S L(\operatorname{rad}(I)) /\{ \pm 1\}$ also acts on $\mathbf{H}$ faithfully and transitively. Hence $\mathbb{E}$ and $\mathbf{H}$ have the structure of homogeneous spaces. These groups act on the bundle $\mathbb{E} \stackrel{\pi}{\rightarrow} H$ equivariantly.

\section{(3.5) The Central Extension of $\mathrm{Aut}^{+}(\boldsymbol{R})$}

In order to lift the action of $\mathrm{Aut}^{+}(R)$ on $\mathbf{E}$ to $\tilde{\mathbf{E}}$, we need to construct the central extension $\mathrm{Aut}^{+}(R)$ of $\mathrm{Aut}^{+}(R)$. In Definition 3.6, we define the central extension $\tilde{O_{\tilde{\mathrm{E}}}^{+}}(F, I)$ of $O^{+}(F, I) /\{ \pm 1\}$. The central extension $\tilde{O^{+}}(F, I)$ of $\mathrm{O}^{+}(F, I)$ is defined in Definition 3.7, Proposition 3.8. $\mathrm{Aut}^{+}(R)$ will be defined in Definition 3.9 as a subgroup of $\tilde{O^{+}}(F, I)$.

First, we define an automorphic factor. In (2.6) Remark, we looked at $b$, an element of the $\mathbb{Z}$-basis of $\operatorname{rad}(I) \cap Q(R)$ (introduced in (2.2.1)), as a coordinate function $\tau$ of $\mathbf{H}$. Since $b$ is unique up to adding $m \times a(m \in \mathbb{Z})$ 
((2.2) Remark), $\tau$ is unique up to adding $m \in \mathbf{Z}$. Thus $d \tau$ is a well defined 1 -form on $\mathbf{H}$.

By the Remark in (3.4), $h \in S L(\operatorname{rad}(I)) /\{ \pm 1\}$ induces an isomorphism of H. Thus $\tau \circ h$ is a function on $\mathbf{H}$ which is unique up to adding $m \in \mathbb{Z}$. Therefore $d(\tau \circ h)$ is a well defined 1 -form on $\mathbf{H}$. By the identity $d(\tau \circ h)=$ $\frac{d(\tau \circ h)}{d \tau} d \tau, \frac{d(\tau \circ h)}{d \tau}$ is a well defined function on $\mathbf{H}$.

If $h \in S L(\operatorname{rad}(I)) /\{ \pm 1\}$ is represented by a matrix

$$
\left(\begin{array}{ll}
p & q \\
r & s
\end{array}\right)^{-1} \in S L(2, \mathbf{Z})
$$

for a $\mathbf{Z}$-basis $a, b \in \operatorname{rad}(I)$ as above, then $\frac{d(\tau \circ h)}{d \tau}$ is represented by $(r \tau+s)^{-2}$. We call the function $\frac{d(\tau \circ h)}{d \tau}$ an automorphic factor for $h$.

Now, we consider the following conditions on a holomorphic bundle isomorphism $f: \tilde{\mathbf{E}} \rightarrow \tilde{\mathbf{E}}$ with respect to the affine bundle structure $\tilde{\pi}: \tilde{\mathbf{E}} \rightarrow \mathbf{E}$.

Conditions. For a holomorphic bundle map $f: \tilde{\mathbf{E}} \rightarrow \tilde{\mathbf{E}}$, there exists the unique $f_{0} \in O^{+}(F, I) /\{ \pm 1\}$, such that

$$
\begin{aligned}
& \text { 1) } \tilde{\pi} \circ f=f_{0} \circ \tilde{\pi} \text { on } \tilde{\mathbf{E}}, \\
& \text { 2) } f^{*} \tilde{I}_{\mathbf{E}}^{*}=\frac{d\left(\tau \circ \rho\left(f_{0}\right)\right)}{d \tau} \tilde{I}_{\mathbf{E}}^{*},
\end{aligned}
$$

where $\tilde{I}_{\mathbf{E}}^{*}$ is the holomorphic metric on $\operatorname{Der}_{\overline{\mathbf{E}}}$ defined in (2.8.5).

Definition 3.6.

$\widetilde{O_{\tilde{\mathbf{E}}}^{ \pm}}(F, I):=\{f: \tilde{\mathbf{E}} \rightarrow \tilde{\mathbf{E}}$ holomorphic bundle isomorphism of $\tilde{\pi}: \tilde{\mathbf{E}} \rightarrow \mathbb{E} ;$ which satisfies the above conditions (3.5.1), (3.5.2)\}.

From the condition (3.5.1), we can define the homomorphism:

$$
\psi: \tilde{O_{\widetilde{\mathbf{E}}}^{+}}(F, I) \rightarrow O^{+}(F, I) /\{ \pm 1\} \text {. }
$$

Since $\frac{d\left(\tau \circ \rho\left(f_{0}\right)\right)}{d \tau}$ satisfies the cocycle condition with respect to the group $S L(\operatorname{rad}(I)) /\{ \pm 1\}, \tilde{O_{\overline{\mathbf{E}}}^{+}}(F, I)$ has a group structure by composition.

Remark. The Weyl group $\tilde{W}_{R}$ acts on the space $\tilde{\mathbf{E}}$ faithfully satisfying the above condition (3.5.1)(3.5.2), thus we have the natural inclusion map $l: \tilde{W}_{R} \rightarrow \tilde{O_{\tilde{\mathbf{E}}}^{+}}(F, I)$.

After the preparations above, we define the central extension $\widetilde{O^{+}}(F, I)$ of $O^{+}(F, I)$. Let $\varphi$ be the natural projection of $O^{+}(F, I) \rightarrow O^{+}(F, I) /\{ \pm 1\}$. 
Definition 3.7.

$$
\widetilde{O^{+}}(F, I):=\left\{(x, y) \in \widetilde{O_{\tilde{\mathbf{E}}}^{+}}(F, I) \times O^{+}(F, I) \mid \psi(x)=\varphi(y)\right\} .
$$

$\widetilde{O^{+}}(F, I)$ has a group structure in a natural way. We call the next two projections $p_{1}$ and $p_{2}$ :

$$
\begin{aligned}
& p_{1}: \tilde{O^{+}}(F, I) \rightarrow \tilde{O_{\tilde{\mathbf{E}}}^{+}}(F, I) \\
& (x, y) \mapsto(x), \\
& p_{2}: \tilde{O^{+}}(F, I) \rightarrow O^{+}(F, I) \\
& (x, y) \mapsto(y) .
\end{aligned}
$$

We define the action of $\tilde{O^{+}}(F, I)$ on $\tilde{\mathbb{E}}$ through $p_{1}$.

Remark. We have a natural embedding:

$$
\begin{aligned}
\tilde{W}_{R} & \rightarrow \tilde{O^{+}}(F, I) \\
g & \mapsto\left(l(g),\left.g\right|_{F}\right) .
\end{aligned}
$$

Hereafter we regard $\tilde{W}_{R}$ as a subgroup of $\tilde{O}^{+}(F, I)$ by the above homomorphism (3.5.6).

Proposition 3.8. $\tilde{O^{+}}(F, I)$ is a central extension of $O^{+}(F, I)$. We have the following diagram:

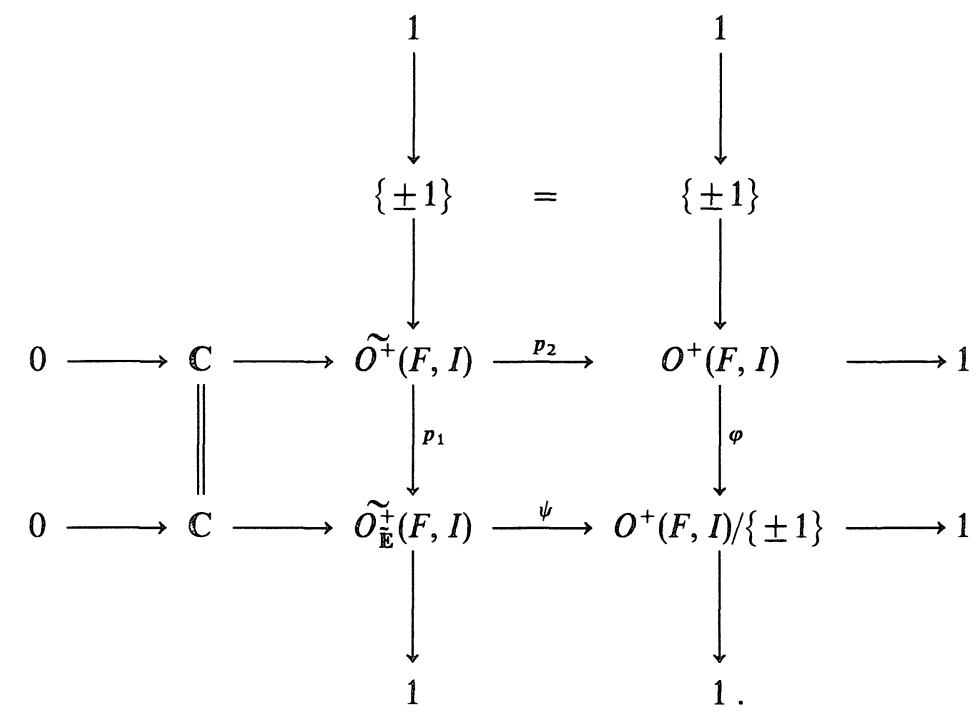

Proof. We should only prove the exactness of the third row sequence. The other part of the proof of the diagram (3.5.7) is automatic. 
We choose a trivialization of the affine bundle $\tilde{\mathbf{E}}$ introduced in (2.7.3):

$$
(\tilde{\pi}, \tilde{\lambda}): \tilde{\mathbf{E}} \simeq \mathbf{E} \times \mathbf{C} .
$$

For all $f_{0} \in O^{+}(F, I) /\{ \pm 1\}$, we must study the existence and the ambiguity of the function $g(x, t)$ such that

$$
\begin{aligned}
\mathbf{E} \times \mathbf{C} & \mapsto \mathbf{E} \times \mathbf{C} \\
(x, t) & \mapsto\left(f_{0}(x), g(x, t)\right),
\end{aligned}
$$

is an element of $\tilde{O_{\overline{\mathbf{E}}}^{+}}(F, I)$. The projection $p: F \rightarrow F / \operatorname{rad}(I)$ induces the homomorphism $p: O^{+}(F, I) \rightarrow G L(F / \operatorname{rad}(I))$. The image $p\left(O^{+}(F, I)\right)$ is $O(F / \operatorname{rad}(I), \bar{I})$, where $\bar{I}$ is the bilinear form on $F / \operatorname{rad}(I)$ induced from $I$. This enables us to reduce the condition $f^{*} \tilde{I}_{\mathbf{E}}^{*}=\frac{d\left(\tau \circ \rho\left(f_{0}\right)\right)}{d \tau} \tilde{I}_{\mathbf{E}}^{*}$ (3.5.2) to a total differential equation of the type $d g(x, t)=\omega$, where $\omega$ is a holomorphic 1-form.

The action $\mathrm{Aut}^{+}(R)$ on $\mathbf{E}$ is a linear fractional transformation, therefore we find that $\omega$ is a closed 1 -form. Since $\tilde{\mathbf{E}}$ is a simply connected space, we know that $g(x, t)$ always exists, and that the ambiguity of $g(x, t)$ is described by $\mathbf{C}$, the translation of the affine bundle $\tilde{\pi}: \tilde{\mathbf{E}} \rightarrow \mathbf{E}$.

$g(x, t)$ has the form of $t+g_{1}(x)$, therefore the condition (3.5.2) also implies that the map (3.5.9) becomes a bundle isomorphism of $\tilde{\pi}: \tilde{\mathbf{E}} \rightarrow \mathbf{E}$ as an affine bundle.

Q.E.D.

Remark 1. Y. Namikawa told the author that this technique of extending the group action was used in the theory of symmetric domains of type IV (cf. [10] p.148).

Remark 2. $\tilde{O^{+}}(F, I)$ acts on $\tilde{\mathbf{E}}$ transitively, therefore $\tilde{\mathbf{E}}$ has the structure of a homogeneous space.

Definition 3.9. The central extension of $\operatorname{Aut}^{+}(R)$ is defined as follows:

$$
\operatorname{Aut}^{+}(R):=\left(p_{2}\right)^{-1}\left(\operatorname{Aut}^{+}(R)\right) \text {. }
$$

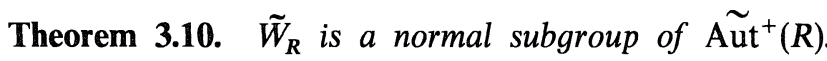

Proof. For any $g \in \tilde{O^{+}}(F, I)$, we shall prove

$$
g \tilde{w}_{\alpha} g^{-1}=\tilde{w}_{\bar{g} \alpha},
$$

where $\alpha \in R$ and $\bar{g}=p_{2}(g) \in O^{+}(F, I)$. If the equation (3.5.10) holds, then we have the above theorem by considering only the cases $g \in \tilde{A u t}^{+}(R)$.

If we apply the homomorphism $p_{2}$ to both sides of the equation (3.5.10), then the equation $p_{2}\left(g \tilde{w}_{\alpha} g^{-1}\right)=p_{2}\left(\tilde{w}_{\bar{g} \alpha}\right)$ holds by the same calculation as in the proof of Proposition 3.2. Hence we should only prove the equation: 


$$
p_{1}\left(g \tilde{w}_{\alpha} g^{-1}\right)=p_{1}\left(\tilde{w}_{\bar{g} \alpha}\right) .
$$

In other words, we should only prove the equation (3.5.10) on $\tilde{\mathbb{E}}$.

First, we call the inverse image of the subgroup $O(F, \operatorname{rad}(I)) \subset O^{+}(F, I) /$ $\{ \pm 1\}$ of the homomorphism (3.5.3) $\left.\psi: \widetilde{O_{\tilde{\mathbf{E}}}^{ \pm}}(F, I) \rightarrow O^{+}(F, I) /\{ \pm 1\}, \tilde{O(F}, \operatorname{rad}(I)\right)$.

This leads to the following diagram:

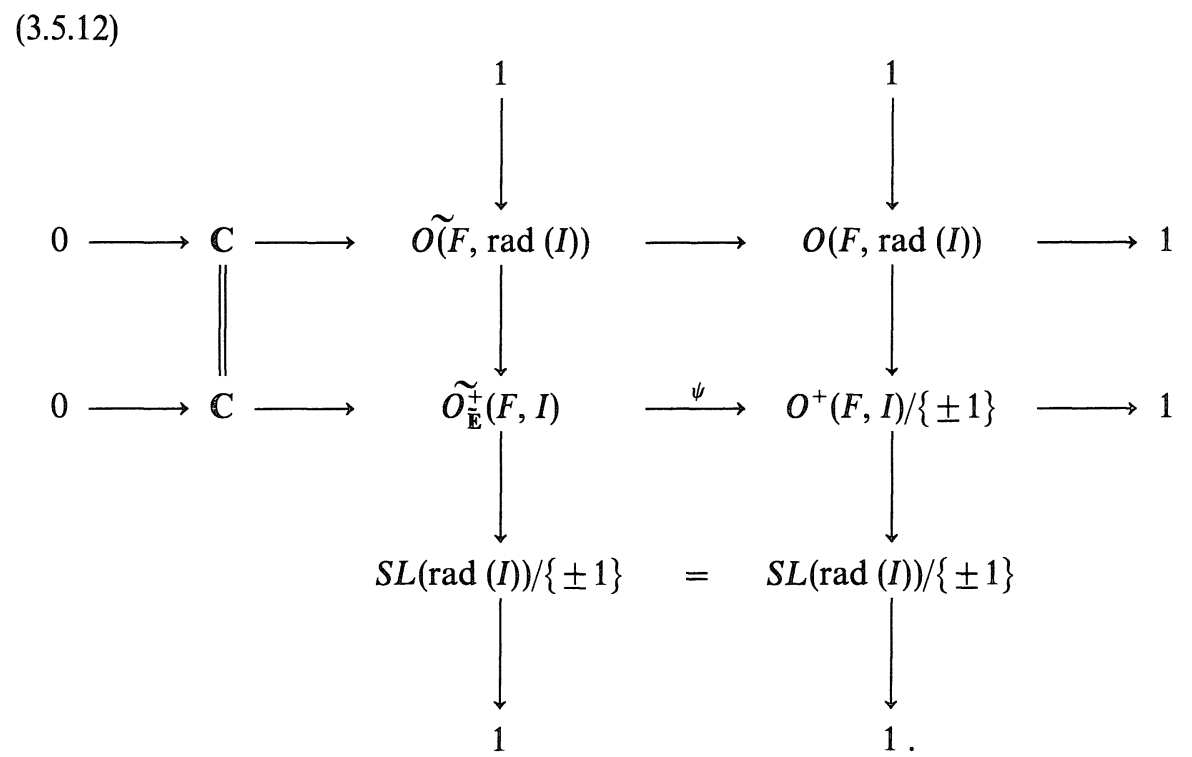

By this diagram, we can reduce the proof to two parts: 1. (3.5.11) holds for $g \in \widetilde{O(F}, \operatorname{rad}(I))$. 2. (3.5.11) holds for some lifting of $S L(\operatorname{rad}(I)) /\{ \pm 1\}$ to $\widetilde{O_{\tilde{\mathbb{E}}}^{+}}(F, I)$.

Lemma 3.11. (3.5.11) holds for $g \in \tilde{O(F}, \operatorname{rad}(I))$.

Proof. We prepare some notations. $F_{\mathbf{C}}:=F \otimes_{\mathbb{R}} \mathbf{C} . \quad \tilde{F}_{\mathbf{C}}:=\tilde{F} \otimes_{\mathbb{R}} \mathbb{C} . \quad \mathbb{R} \subset$ $\mathbb{C}$ induces $F \subset F_{\mathbf{C}} . \quad I_{\mathbf{C}}:=$ the $\mathbf{C}$-linear extension of $I$, which gives a bilinear form on $F_{\mathbf{C}}$. $\tilde{I}_{\mathbf{C}}:=$ the $\mathbb{C}$-linear extension of $\tilde{I}$, which gives a bilinear form on $\tilde{F}_{\mathrm{C}}$. We define $\left.\widehat{O(F}, \operatorname{rad}(I)\right)$ as follows:

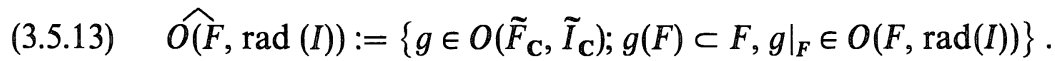

Since $g \in \widehat{O(F}, \operatorname{rad}(I))$ satisfies the conditions of $\widetilde{O(F}, \operatorname{rad}(I))$, we have the 
homomorphism:

$$
\widehat{O(F}, \operatorname{rad}(I)) \rightarrow \widetilde{O(F}, \operatorname{rad}(I))
$$

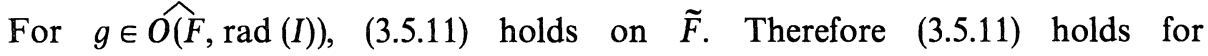
the image of the homomorphism (3.5.13). We show that $\widetilde{O(F, \operatorname{rad}(I))=}$ $\widehat{O(F}, \operatorname{rad}(I))$. Since $\operatorname{dim}_{\mathbf{C}} \operatorname{rad}(\tilde{I})=1$, we have the following commutative diagram:

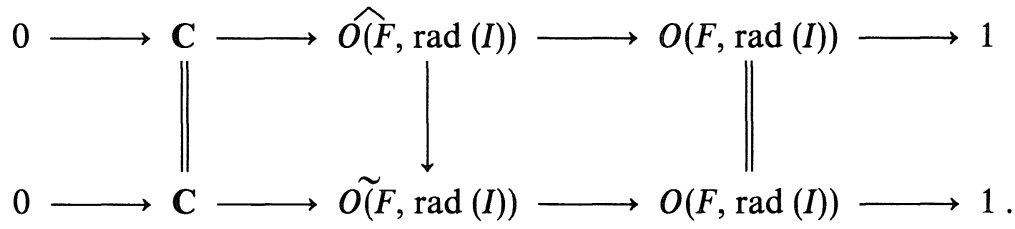

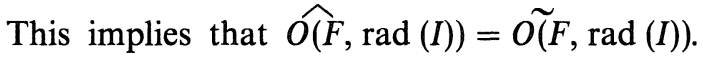

Lemma 3.12. (3.5.11) holds for some lifting of $S L(\operatorname{rad}(I)) /\{ \pm 1\}$ to $\widetilde{O_{\tilde{\mathbf{E}}}^{+}}(F, I)$.

Proof. Fixing a basis (see (2.1)), we have a trivialization:

$$
\begin{aligned}
& \tilde{\mathbf{E}} \simeq \quad \mathbf{H} \times \mathbf{C}^{l} \times \mathbf{C} \\
& (x) \mapsto\left(b(x), \alpha_{1}(x), \ldots, \alpha_{l}(x), \lambda(x)\right) .
\end{aligned}
$$

We denote the elements of $\mathbf{H} \times \mathbf{C}^{l} \times \mathbf{C}$ by $(\tau, z, t)$. One lifting of $S L(\operatorname{rad}(I))$ $\ni\left(\begin{array}{ll}p & q \\ r & s\end{array}\right)^{-1}$ is as follows:

$$
(\tau, z, t) \mapsto\left(\frac{p \tau+q}{r \tau+s}, \frac{z}{r \tau+s}, t+\frac{r\langle z, z\rangle}{2(r \tau+s)}\right),
$$

where $\langle$,$\rangle is the \mathbf{C}$-bilinear form on $\mathbf{C}^{l}$ induced from $\tilde{I}$. (3.5.11) can be proved for this lifting by explicit calculation.

Q.E.D of Lemma 3.12 .

Q.E.D of Theorem 3.10 .

Consequently, we obtain the following diagram: 


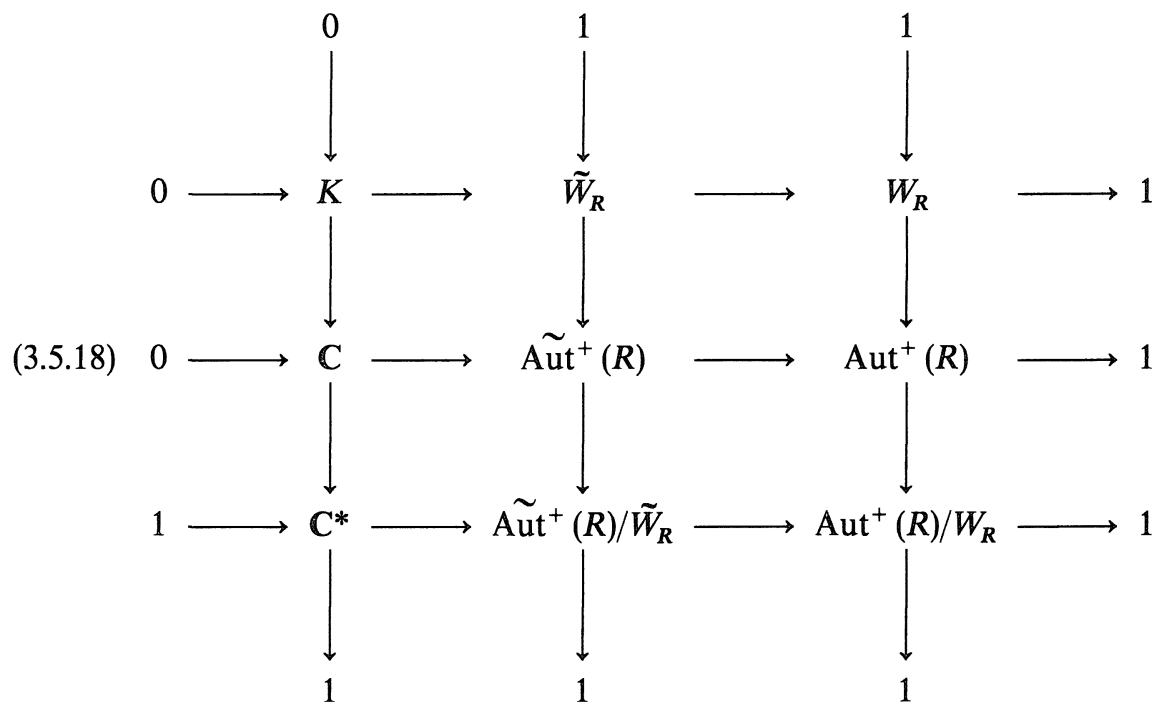

where $C^{*}$ was normalized such that $\alpha \in C^{*}$ acts on $S_{k}$ as the multiplication by $\alpha^{k}$. $\operatorname{Aut}^{+}(R) / \tilde{W}_{R}$ contains $C^{*}$ as its center, thereby $\tilde{A u t}^{+}(R) / \tilde{W}_{R}$ acts on $S^{W}$ as a degree-preserving transformation.

\section{§4. The Action of $\mathrm{Aut}^{+}(R)$ on the Flat Theta Invariants}

In [16], Saito introduced the non-degenerate holomorphic metric $J^{*}$ on the submodule $\mathscr{F} \subset \operatorname{Der}_{s^{w}}$, and proved that the Levi-Civita connection $\nabla$ on $\mathscr{F}$ with respect to $J^{*}$ is integrable. He called the $\tilde{W}_{R}$ invariants associated to $J^{*}$, the flat theta invariants. In this section, we study the action of $\tilde{A u t}^{+}(R)$ on the holomorphic metric $J^{*}$, and in the Theorem 4.7, we shall describe the action of $\mathrm{Aut}^{+}(R)$ on the flat theta invariants explicitly.

In the rest of this paper, we assume that the codimension of the marked extended affine root system $(R, G)$ equals one. (The notion of codimension was introduced in $\S 2$.)

\section{(4.1) Normalized Lowest Degree Vector Field and the Holomorphic Metric $J^{*}$}

In Theorem 2.2, it was shown that there exist algebraically free generators of the algebra $S^{W}: \Theta_{0}, \cdots, \Theta_{l}$ over $\Gamma\left(\mathbf{H}, \mathcal{O}_{\mathbf{H}}\right)$. In the $S^{W}$-graded module $\operatorname{Der}_{S^{w}}$, the module of the lowest degree vector fields is a free $\Gamma\left(\mathbf{H}, \mathcal{O}_{\mathbf{H}}\right)$-module of rank 1 (= codimension) generated by $\frac{\partial}{\partial \Theta_{l}}$.

Multiplying by a function $h \in \Gamma\left(\mathbf{H}, \mathcal{O}_{\mathbf{H}}^{*}\right)$, if necessary, we can take a highest degree generator $\Theta_{l}$ which satisfies 


$$
\frac{\partial^{2}}{\partial \Theta_{l}^{2}} \tilde{I}_{W}\left(d \Theta_{l}, d \Theta_{l}\right)=0 .
$$

By (4.1.1), $\frac{\partial}{\partial \Theta_{l}}$ is normalized up to a constant factor.

Hereafter we fix $\Theta_{0}, \cdots, \Theta_{l}$ such that $\Theta_{l}$ satisfies the condition (4.1.1). We can define

$$
T:=\left\{f \in S^{W} \mid \frac{\partial}{\partial \Theta_{l}} f=0\right\},
$$

$$
\begin{aligned}
\operatorname{Der}_{T} & :=\text { the module of } \mathbf{C} \text {-derivations of the algebra } T, \\
\Omega_{T}^{1} & :=\text { the module of } 1 \text {-forms for the algebra } T, \\
\mathscr{G} & :=\left\{\xi \in \operatorname{Der}_{S^{w}} \mid\left[\frac{\partial}{\partial \Theta_{l}}, \xi\right]=0\right\}, \\
\mathscr{F} & :=\left\{\omega \in \Omega_{S^{w}}^{1} \mid L_{\partial / \partial \Theta_{l}} \omega=0\right\},
\end{aligned}
$$

where $L_{\partial / \partial \Theta_{l}}$ means the Lie derivative with respect to the vector field $\frac{\partial}{\partial \Theta_{l}}$. There is a canonical inclusion $\Omega_{T}^{1} \subset \mathscr{F}$. By the above generators $\tau, \Theta_{0}, \cdots, \Theta_{l}$, we can represent $T, \operatorname{Der}_{T}, \Omega_{T}^{1}, \mathscr{G}, \mathscr{F}$ as follows:

$$
\begin{aligned}
T & =\Gamma\left(\mathbf{H}, \mathcal{O}_{\mathbf{H}}\right)\left[\Theta_{0}, \cdots, \Theta_{l-1}\right], \\
\operatorname{Der}_{T} & =T \frac{\partial}{\partial \tau} \oplus \bigoplus_{i=0}^{l-1} T \frac{\partial}{\partial \Theta_{i}}, \\
\Omega_{T}^{1} & =T d \tau \oplus \bigoplus_{i=0}^{l-1} T d \Theta_{i}, \\
\mathscr{G} & =T \frac{\partial}{\partial \tau} \oplus \bigoplus_{i=0}^{l} T \frac{\partial}{\partial \Theta_{i}}, \\
\mathscr{F} & =T d \tau \oplus \bigoplus_{i=0}^{l} T d \Theta_{i} .
\end{aligned}
$$

The pairing $\operatorname{Der}_{S^{w}} \times \Omega_{S^{W}}^{1} \rightarrow S^{W}$ induces the complete pairing $\mathscr{G} \times \mathscr{F} \rightarrow T$, so $\mathscr{G}$ is a $T$-dual module of $\mathscr{F}$.

We define a $T$-bilinear form:

$$
\begin{aligned}
J^{*}: \mathscr{F} \times \mathscr{F} & \rightarrow T, \\
\omega_{1} \times \omega_{2} & \mapsto \frac{\partial}{\partial \Theta_{l}} \tilde{I}_{W}\left(\omega_{1}, \omega_{2}\right) .
\end{aligned}
$$

The value $\frac{\partial}{\partial \Theta_{l}} \tilde{I}_{W}\left(\omega_{1}, \omega_{2}\right)$ belongs to $T$ by the condition (4.1.1). Then the next 
important fact was shown by the Coxeter transformation theory for the extended affine root system.

Proposition 4.1 ([16, p.49]). The $T$ bilinear form $J^{*}$ is non-degenerate.

Accordingly, we can also define the $T$-isomorphism:

$$
J *: \mathscr{F} \rightarrow \mathscr{G}
$$

$$
\omega \mapsto J^{*}(\omega, \cdot),
$$

and the $T$-bilinear form $J$ on $\mathscr{G}$ :

$$
\begin{aligned}
J: \mathscr{G} \times \mathscr{G} & \rightarrow T \\
\delta_{1} \times \delta_{2} & \mapsto J^{*}\left(J^{*}\left(\delta_{1}\right), J^{*}\left(\delta_{2}\right)\right) .
\end{aligned}
$$

(4.2) The Action of $\mathrm{Aut}^{+}(\boldsymbol{R})$ on the Holomorphic Metric $J^{*}$

We shall study the action of $\mathrm{Aut}^{+}(R)$ on $J^{*}$. We recall that $\tilde{\mathrm{uut}}^{+}(R)$ acts on $\tilde{\mathbf{E}}$ through $p_{1}$ :

$$
\begin{aligned}
\left.p_{1}\right|_{\text {Aut }^{+}(R)}: \tilde{\operatorname{Aut}}^{+}(R) & \rightarrow \tilde{O_{\tilde{\mathbb{E}}}^{+}}(F, I), \\
g & \mapsto p_{1}(g) .
\end{aligned}
$$

Proposition 4.2. The action of $\mathrm{Aut}^{+}(R)$ on $\frac{\partial}{\partial \Theta_{l}}$ is described as follows:

$$
\left(p_{1}\left(g^{-1}\right)\right)_{*}\left(\frac{\partial}{\partial \Theta_{l}}\right)=\chi(g) \frac{\partial}{\partial \Theta_{l}}, \quad\left(g \in \tilde{A u t}^{+}(R)\right)
$$

where $\chi$ is a group homomorphism:

$$
\chi: \tilde{A u t}^{+}(R) \rightarrow C^{*} .
$$

Proof. Since the action of $\operatorname{Aut}^{+}(R)$ on $\operatorname{Der}_{S^{w}}$ is a degree preserving transformation, there exists a non-vanishing holomorphic function $f(\tau)$ on $\mathbf{H}$ satisfying $\left(p_{1}\left(g^{-1}\right)\right)_{*}\left(\frac{\partial}{\partial \Theta_{l}}\right)=f(\tau) \frac{\partial}{\partial \Theta_{l}}$ for $g \in \tilde{A u t}^{+}(R)$. We only have to prove that $f(\tau)=$ const. $\in \mathbb{C}$.

First, we notice that

$$
p_{1}(g)^{*} \Theta_{l}=f^{-1}(\tau) \Theta_{l}+u
$$

for some $u \in T$. If $v \in T$ and $h(\tau) \in \Gamma\left(\mathbf{H}, \mathcal{O}_{\mathbf{H}}\right)$, then $\tilde{I}_{W}(d v, d v)$ and $\tilde{I}_{W}\left(d\left(h(\tau) \Theta_{l}\right), d v\right)$ have at most degree 1 with respect to $\Theta_{l}$. Thus

$$
\frac{\partial^{2}}{\partial \Theta_{l}^{2}} \tilde{I}_{W}(d v, d v)=0
$$




$$
\frac{\partial^{2}}{\partial \Theta_{l}^{2}} \tilde{I}_{W}\left(d\left(h(\tau) \Theta_{l}\right), d v\right)=0
$$

We recall that

$$
\tilde{I}_{W}\left(d \tau, d \Theta_{l}\right)=\kappa^{-1} \Theta_{l} \quad(2.8 .12)
$$

and that

$$
p_{1}(g) * \tilde{I}_{W}(d \varphi, d \psi)=\left(\frac{d\left(\tau \circ \rho\left(p_{1}(g)\right)\right.}{d \tau}\right)^{-1} \tilde{I}_{W}\left(d\left(p_{1}(g)^{*} \varphi\right), d\left(p_{1}(g)^{*} \psi\right)\right)
$$

We apply $p_{1}(g)$ on the both sides of equality (4.1.1), we have

$$
\begin{aligned}
0= & p_{1}(g)^{*}\left[\frac{\partial^{2}}{\partial \Theta_{l}^{2}} \tilde{I}_{W}\left(d \Theta_{l}, d \Theta_{l}\right)\right] \\
= & f^{2}(\tau)\left(\frac{d\left(\tau \circ \rho\left(p_{1}(g)\right)\right.}{d \tau}\right)^{-1} \frac{\partial^{2}}{\partial \Theta_{l}^{2}} \tilde{I}_{W}\left(d\left(f^{-1}(\tau) \Theta_{l}+h\right), d\left(f^{-1}(\tau) \Theta_{l}+h\right)\right) \\
= & f^{2}(\tau)\left(\frac{d\left(\tau \circ \rho\left(p_{1}(g)\right)\right.}{d \tau}\right)^{-1} \frac{\partial^{2}}{\partial \Theta_{l}^{2}} \tilde{I}_{W}\left(d\left(f^{-1}(\tau) \Theta_{l}\right), d\left(f^{-1}(\tau) \Theta_{l}\right)\right) \\
= & f^{2}(\tau)\left(\frac{d\left(\tau \circ \rho\left(p_{1}(g)\right)\right.}{d \tau}\right)^{-1} \frac{\partial^{2}}{\partial \Theta_{l}^{2}}\left[\left(f^{-1}(\tau)\right)^{2} \tilde{I}_{W}\left(d \Theta_{l}, d \Theta_{l}\right)\right. \\
& \left.+2 f^{-1}(\tau) \Theta_{l} \tilde{I}_{W}\left(d\left(f^{-1}\right), d \Theta_{l}\right)+\Theta_{l}^{2} \tilde{I}_{W}\left(d\left(f^{-1}\right), d\left(f^{-1}\right)\right)\right] \\
= & 2 f^{2}(\tau)\left(\frac{d\left(\tau \circ \rho\left(p_{1}(g)\right)\right.}{d \tau}\right)^{-1} \frac{\partial f}{\partial \tau} \frac{1}{\kappa f^{3}(\tau)} .
\end{aligned}
$$

Since $f(\tau),\left(\frac{d\left(\tau \circ \rho\left(p_{1}(g)\right)\right.}{d \tau}\right)^{-1}$ don't vanish, $f(\tau)$ must be a constant. Q.E.D.

By Proposition 4.2, $\operatorname{Aut}^{+}(R)$ acts on $T, \mathscr{G}$ and $\mathscr{F}$.

Therefore the automorphism group $\tilde{A u t}^{+}(R)$ acts also on $J^{*}$ in a natural way.

Proposition 4.3. The action of $\tilde{A u t}^{+}(R)$ on $J^{*}$ is described as follows:

$$
p_{1}(g)^{*}\left[J^{*}\left(\omega_{1}, \omega_{2}\right)\right]=\chi(g)\left(\frac{d\left(\tau \circ \rho\left(p_{1}(g)\right)\right.}{d \tau}\right)^{-1} J^{*}\left(p_{1}(g)^{*} \omega_{1}, p_{1}(g)^{*} \omega_{2}\right)\left(g \in \mathrm{Aut}^{+}(R)\right)
$$
for $\omega_{1}, \omega_{2} \in \mathscr{F}$.

Proof. It's easy to see from Proposition 4.2, and the transformation property (3.5.2) of $\tilde{I}$.

Q.E.D. 


\section{(4.3) The Levi Civita Connection for the Holomorphic Metric $J^{*}$}

We define the Levi Civita connection $\nabla$ on $\mathscr{F}$ with respect to $J^{*}$. Then the Proposition 4.4 asserts that the connection $\nabla$ is integrable.

Proposition 4.4 ([16, p.58]). There exists a unique torsion-free, integrable, metric (w.r.t. $\left.J^{*}\right)$ connection $\nabla$ on $\mathscr{F}$ as a T-module:

$$
\nabla: \operatorname{Der}_{T} \times \mathscr{F} \rightarrow \mathscr{F},
$$

$$
(\delta, \omega) \mapsto\left(\nabla_{\delta} \omega\right) .
$$

I.e.

0) The map $\nabla_{\delta} \omega$ is T-linear in $\delta$ and satisfies the Leibniz rule:

$$
\nabla_{\delta}(f \omega)=\delta(f) \omega+f \nabla_{\delta} \omega \quad \text { for } f \in T .
$$

1) (integrability) For $\forall \delta, \xi \in \operatorname{Der}_{T}$,

$$
\left[\nabla_{\delta}, \nabla_{\xi}\right]=\nabla_{[\delta, \xi]} .
$$

2) (torsion-freeness) The following diagram commutes:

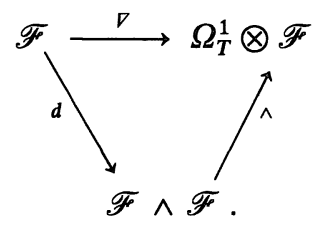

3) (metric) For $\delta \in \operatorname{Der}_{T}$ and $\omega_{1}, \omega_{2} \in \mathscr{F}$,

$$
\delta J^{*}\left(\omega_{1}, \omega_{2}\right)=J^{*}\left(\nabla_{\delta} \omega_{1}, \omega_{2}\right)+J^{*}\left(\omega_{1}, \nabla_{\delta} \omega_{2}\right) .
$$

Remark. The torsion-freeness implies that a horizontal section $\omega \in \mathscr{F}$ (with respect to $\nabla$ ) is a closed form.

Proposition 4.5. The action of $\tilde{A u t}^{+}(R)$ on $\nabla_{\delta} \omega$ is described as follows:

$$
p_{1}(g)^{*}\left(\nabla_{\delta} \omega\right)=\nabla_{\tilde{\delta}} \tilde{\omega}+\frac{1}{2 f}\left[(\tilde{\delta} f) \tilde{\omega}-J^{*}(\tilde{\omega}, d f)\left(J^{*}\right)^{-1}(\tilde{\delta})+\langle\tilde{\delta}, \tilde{\omega}\rangle d f\right]
$$

where $\tilde{\delta}=p_{1}\left(g^{-1}\right)_{*} \delta, \tilde{\omega}=p_{1}(g)^{*} \omega, f=\left(\frac{d\left(\tau \circ \rho\left(p_{1}(g)\right)\right.}{d \tau}\right)^{-1}$ and $\delta \in \operatorname{Der}_{T}, \omega \in \mathscr{F}$.

Proof. This follows from Proposition 4.3 and the formula:

$$
\begin{aligned}
2 J^{*}\left(\nabla_{\delta} \omega_{1}, \omega_{2}\right)= & \delta J^{*}\left(\omega_{1}, \omega_{2}\right)+\left\langle\omega_{1},\left[J^{*}\left(\omega_{2}\right), \delta\right]\right\rangle-J^{*}\left(\omega_{2}\right)\left\langle\delta, \omega_{1}\right\rangle \\
& -\left\langle\left[J^{*}\left(\omega_{1}\right), J^{*}\left(\omega_{2}\right)\right],\left(J^{*}\right)^{-1}(\delta)\right\rangle \\
& -\left\langle\omega_{2},\left[J^{*}\left(\omega_{1}\right), \delta\right]\right\rangle+J^{*}\left(\omega_{1}\right)\left\langle\delta, \omega_{2}\right\rangle,
\end{aligned}
$$

for $\delta \in \operatorname{Der}_{T}, \omega_{1}, \omega_{2} \in \mathscr{F}$.

Q.E.D. 


\section{(4.4) Modular Property for the Flat Theta Invariants}

We rewrite the degrees $0, \tilde{m}_{0}, \cdots, \tilde{m}_{l}$ of $\tau, \Theta_{0}, \cdots, \Theta_{l}$ as follows:

$$
\begin{aligned}
0 & =m_{0,1}=\cdots=m_{0, n_{0}}<m_{1,1}=\cdots=m_{1, n_{1}}<m_{2,1}=\cdots \\
& =m_{2, n_{2}}<\cdots<m_{k, 1}=\cdots=m_{k, n_{k}}<m_{k+1,1}=\cdots=m_{k+1, n_{k+1}},
\end{aligned}
$$

such that $\tilde{m}_{i}=m_{p, q}$ when $i=q+\sum_{\substack{p=0 \\ j=0}} n_{j}$. By the assumption, codimension = $1, n_{k+1}=1$ and the duality of the exponents holds for $\operatorname{cod}(R, G)=1$, i.e.

$$
n_{i}=n_{k+1-i} \quad(0 \leq i \leq k+1) .
$$

Hereafter we fix one $\mathbf{Z}$ basis $a, b \in \operatorname{rad}(I) \cap Q(R)(a \in G \cap Q(R))$, thereby we also fix the coordinate function $\tau \in \Gamma\left(\mathbf{H}, \mathcal{O}_{\mathbf{H}}\right)$. We represent $\rho \circ \pi(g) \in \Gamma^{+}$ by a matrix

$$
((\rho \circ \pi(g)) b,(\rho \circ \pi(g)) a)=(b, a)^{t}\left(\begin{array}{ll}
p & q \\
r & s
\end{array}\right)^{-1} .
$$

Theorem 4.6 (flat theta invariants $[16, \mathrm{p} .63]$ ). In the module $S^{W}$, there exists a unique complex graded vector space $V$ of rank $l+2$, whose weights are $0=m_{0,1}, m_{1,1}, \ldots, m_{k+1,1}$ i.e.

$$
V=\bigoplus_{i=0}^{k+1} V_{i} \quad \text { where } \operatorname{dim} V_{i}=n_{i} \quad(0 \leq i \leq k+1),
$$

such that

1. $\quad V_{0}=\mathbf{C} \cdot \tau$

2. $S^{W}=\Gamma\left(\mathbf{H}, \mathcal{O}_{\mathbf{H}}\right) \otimes_{\mathbf{C}[\tau]} S[V]$, where $S[V]$ is the symmetric tensor algebra of $V$.

3. $d V \subset \Omega_{S^{w}}^{1}$ becomes the set of horizontal sections of $\mathscr{F}$ with respect to the connection $\nabla$. In particular, $d \tau$ and $\kappa J^{*}(d \tau)=\frac{\partial}{\partial \Theta_{l}}$ are horizontal. ( $\kappa$ is the non-zero constant defined in (2.8.12).)

4. $J^{*}$ defines a non-degenerate $\mathbf{C}$-bilinear form on $V$ using the inclusion map: $V \subset d V \subset \Omega_{S^{w}}^{1}$

$$
J^{*}: V \times V \rightarrow \mathbf{C}
$$

in particular $J^{*}$ defines a complete pairing of $V_{i}$ and $V_{k+1-i}(0 \leq i \leq k+1)$ :

$$
J^{*}: V_{i} \times V_{k+1-i} \rightarrow \mathbf{C} .
$$

We call the elements of $V$ the flat theta invariants.

Theorem 4.7 (modular property for the flat theta invariants). The action of $\mathrm{Aut}^{+}(R)$ on the flat theta invariants is as follows: 


$$
p_{1}(g) * \tau=\frac{p \tau+q}{r \tau+s}
$$

$$
p_{1}(g)^{*} v=\frac{1}{r \tau+s} A_{i}\left(g^{-1}\right) v \quad \text { for all } v \in V_{i}, \quad(1 \leq i \leq k)
$$

$$
p_{1}(g)^{*} \hat{\Theta}_{l}=\chi\left(g^{-1}\right)\left[\hat{\Theta}_{l}+\frac{r}{2 \kappa(r \tau+s)} \sum_{i, j=0}^{l-1} J\left(\frac{\partial}{\partial \hat{\Theta}_{i}}, \frac{\partial}{\partial \hat{\Theta}_{j}}\right) \hat{\Theta}_{i} \hat{\Theta}_{j}\right]
$$

Also $A_{i}$ has a duality with respect to $J^{*}$ :

$J^{*}\left(A_{i}\left(g^{-1}\right) v_{i}, A_{k+1-i}\left(g^{-1}\right) v_{k+1-i}\right)=\chi\left(g^{-1}\right) J^{*}\left(v_{i}, v_{k+1-i}\right) \quad$ for all $v_{i} \in V_{i},(1 \leq i \leq k)$

where

1. $A_{i}: \tilde{\mathrm{Aut}}^{+}(R) \rightarrow G L\left(V_{i}\right)$ is a group homomorphism.

2. $\left\{\hat{\Theta}_{0}, \ldots, \hat{\Theta}_{l-1}\right\}$ is the union of a basis of $\bigoplus_{i=1}^{k} V_{i}, \hat{\Theta}_{l}$ is the element of $V_{k+1}$ which satisfies $\frac{\partial}{\partial \Theta_{l}} \hat{\Theta}_{l}=1$, where $\frac{\partial}{\partial \Theta_{l}}$ was defined and fixed by (4.1.1).

3. $\kappa$ is the non-zero constant defined in (2.8.12).

Proof. Let $\omega=d v$ for $v \in V_{i}(i=1, \ldots, k-1)$. Then $\omega$ is a horizontal section of $\nabla$ of degree $<\tilde{m}_{l}$. By the fact that

$$
J^{*}\left(d\left(p_{1}(g)^{*} v\right), d f\right)=0
$$

(since the degree of (L.H.S) is negative) and by Proposition 4.5, we have

$$
\nabla_{\delta} d\left(p_{1}(g)^{*} v\right)+\frac{1}{2 f}\left[(\delta f) d\left(p_{1}(g)^{*} v\right)+\left\langle\delta, d\left(p_{1}(g)^{*} v\right)\right\rangle d f\right]=0
$$

where $f=\left(\frac{d\left(\tau \circ \rho\left(p_{1}(g)\right)\right.}{d \tau}\right)^{-1}=(r \tau+s)^{2}$. We find that $d \sqrt{f}$ is horizontal (Theorem 4.6-1). Thus $\nabla_{\delta} d\left\{\sqrt{f}\left(p_{1}(g)^{*} v\right)\right\}=0$ for all $\delta \in \operatorname{Der}_{T}$. This implies that $\sqrt{f}\left(p_{1}(g)^{*} v\right) \in V_{i}$. A cocycle condition with respect to the group $\tilde{A u t}^{+}(R)$ satisfied by $\sqrt{f}$ implies that $A_{i}$ is a group homomorphism.

The duality (4.4.10) is a direct consequence of the Proposition 4.3 and the equation (4.4.6).

By Theorem $4.6-3$, it is possible to take $\hat{\Theta}_{l} \in V_{k+1}$ such that $\hat{\Theta}_{l}$ satisfies $\frac{\partial}{\partial \Theta_{l}} \hat{\Theta}_{l}=1$.

The element of the automorphism group $g \in \mathrm{Aut}^{+}(R)$ acts on $J$ as follows: 


$$
\left(p_{1}(g)\right)^{*} J=\chi\left(g^{-1}\right)\left(\frac{d\left(\tau \circ \rho\left(p_{1}(g)\right)\right.}{d \tau}\right) J .
$$

Applying (4.4.13), (4.4.8) and (4.4.10) to

$$
\begin{aligned}
J= & \sum_{i, j=0}^{l-1} J\left(\frac{\partial}{\partial \hat{\Theta}_{i}}, \frac{\partial}{\partial \hat{\Theta}_{j}}\right) d \widehat{\Theta}_{i} \otimes d \hat{\Theta}_{j}+J\left(\frac{\partial}{\partial \tau}, \frac{\partial}{\partial \hat{\Theta}_{l}}\right) d \tau \otimes d \hat{\Theta}_{l} \\
& +J\left(\frac{\partial}{\partial \hat{\Theta}_{l}}, \frac{\partial}{\partial \tau}\right) d \hat{\Theta}_{l} \otimes d \tau
\end{aligned}
$$

we get formula (4.4.9) up to addition of constant. The gradation on $T$ kills this ambiguity and we obtain the formula (4.4.9) exactly.

Q.E.D.

\section{Acknowledgement}

I express my deep gratitude to Prof. Kyoji Saito. A series of his works and lectures introduced me to the present studies. I thank Prof. Isao Naruki and Prof. Yukihiko Namikawa for valuable discussions.

\section{References}

[1] Bernstein, J. N. and Schwarzman, O. V., Chevalley's Theorem for complex crystallographic Coxeter groups, Funct. Anal. Appl., 12 (1978), 79-80.

[2] — Chevalley's Theorem for complex crystallographic Coxeter groups and Affine Root systems, Seminar on Supermanifolds 2, edited by Leites, 22, Matem. Inst. Stockholms Univ., 1986.

[3] Kac, V. G. and Peterson, D., Infinite-dimensional Lie algebras, Theta functions, and modular forms, Adv. Math., 53 (1984), 125-264.

[4] Kas, A. and Schlessinger, M., On the versal deformation of a complex space with an isolated singularity, Math. Ann., 196 (1972), 23-29.

[5] Looijenga, E., On the semi-universal deformation of a simple elliptic singularity, Topology, 17 (1978), 23-40.

[6] - - Root Systems and elliptic curves, Invent. Math., 38 (1976), 17-32.

[7] — Invariant theory for generalized root systems, Invent. Math., 61 (1980), 1-32.

[8] Mérindole, J.-Y., Les singularités simples elliptiques, leurs déformations, les surfaces de Del Pezzo et les transformations quadratiques, Ann. Sci. Ec. Norm. Sup. (4), 15 (1982), $17-44$.

[9] MacDonald, I. G., Affine root systems and Dedekind's $\eta$-functions, Invent. Math., 15 (1972), 91-143.

[10] Namikawa, Y., Toroidal Compactification of Siegel Spaces, Lect. Notes in Math., 812, Springer, Berlin-Heidelberg-New York, 1980.

[11] Pinkham, H., Deformation of normal surface singularities with $\mathrm{C}^{*}$ action, Math. Ann., 232 (1978), 65-84.

[12] Pinkham, H., Simple elliptic singularities, Del Pezzo surfaces and Cremona transformations, Proc. Sympo. Pure Math., 30 I (1977), 69-71. 
[13] Saito, K., Einfach-Elliptish Singularitäten, Invent. Math., 23 (1974), 289-325.

[14] — , Period mapping associated to a primitive form, Publ. RIMS Kyoto Univ., 19 (1983), 1231-1264.

[15] — - Extended Affine Root systems I, Publ. RIMS Kyoto Univ., 21 (1985), 75-179.

[16] — Extended Affine Root systems II, Publ. RIMS Kyoto Univ., 26 (1990), 15-78.

[17] Tjurina, G. N., Locally semiuniversal flat deformation of isolated Singularities of complex spaces, Izv. Akad. Nauk SSSR. Ser. Mat., 33 (1969), 1026-1058 (In Russian). 